Supporting Information for

\title{
Chemoenzymatic Semi-Synthesis of Phosphorylated $\alpha$-Synuclein Enables Identification of a Bidirectional Effect on Fibril Formation
}

\author{
Buyan Pan, Elizabeth Rhoades, * and E. James Petersson* \\ Department of Chemistry, University of Pennsylvania \\ 231 South 34th Street, Philadelphia, Pennsylvania 19104-6323, USA. \\ * elizabeth.rhoades@sas.upenn.edu, ejpetersson@sas.upenn.edu
}

\section{Contents:}

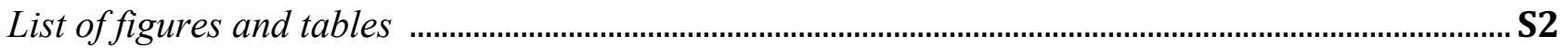

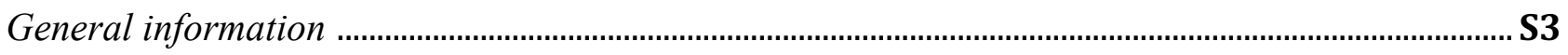

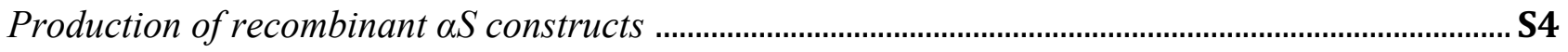

Solid phase peptide synthesis (SPPS) of peptides ..............................................................................................

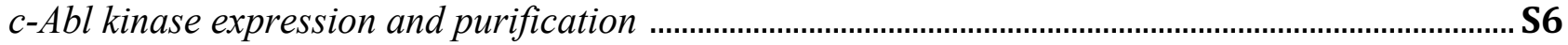

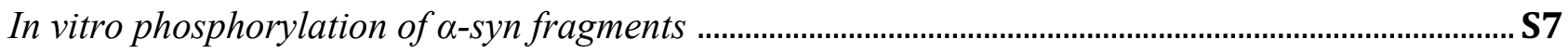

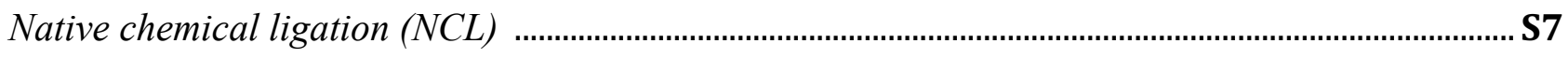

Protein aggregation kinetics and percentage incorporation into fibrils ............................................... S8

Single molecule Förster resonance energy transfer (smFRET) ............................................................S8

Transmission electron microscopy (TEM) ……................................................................................. 8

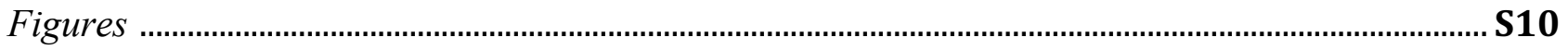

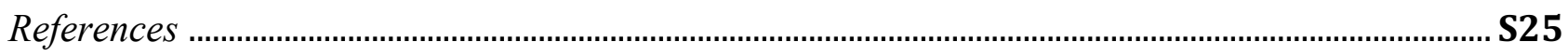




\section{Figures and Tables:}

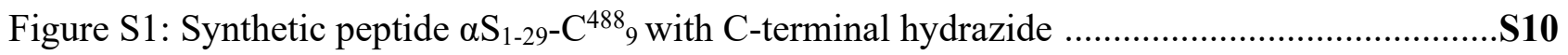

Figure S2. Synthetic peptide $\alpha \mathrm{S}_{30-55}-\mathrm{C}_{30} \mathrm{pY} 39$ with $\mathrm{C}$-terminal hydrazide ..................................S10

Figure S3. Synthetic peptide $\alpha \mathrm{S}_{37-55}-\mathrm{V}^{*}{ }_{37} \mathrm{pY}_{39}$ with $\mathrm{C}$-terminal hydrazide ................................S11

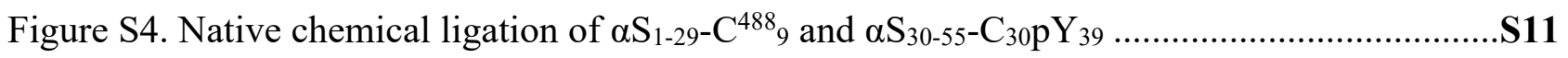

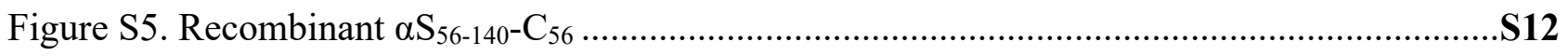

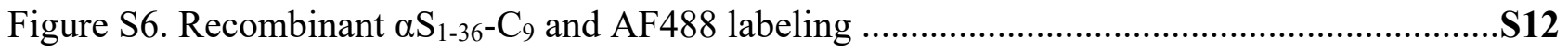

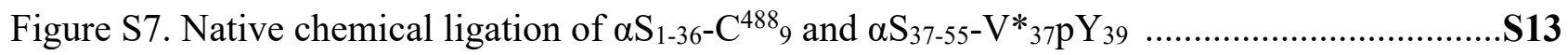

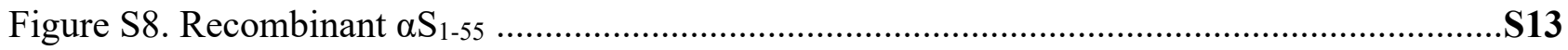

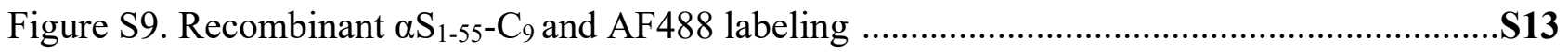

Figure S10. In vitro phosphorylation of $\alpha \mathrm{S}_{1-55}$ by $\mathrm{cAbl}$ kinase ..............................................S14

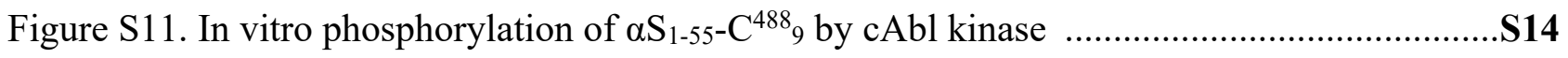

Figure S12. Recombinant $\alpha \mathrm{S}_{56-140}-\mathrm{C}_{56} \pi_{72}$ and AF594 labeling ...........................................S15

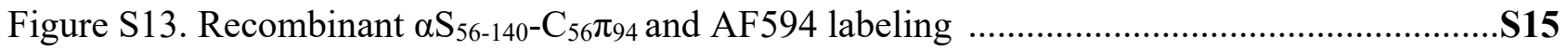

Figure S14. Recombinant $\alpha \mathrm{S}_{56-140}-\mathrm{C}_{56} \pi_{136}$ and AF594 labeling .............................................S16

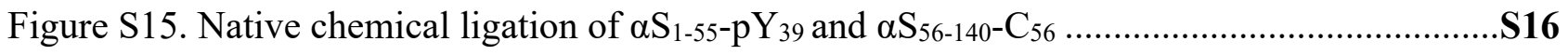

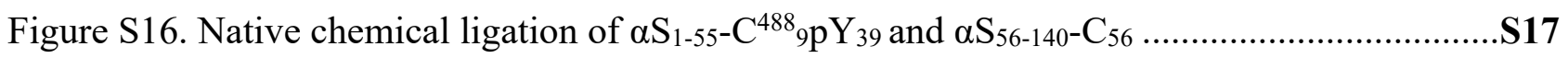

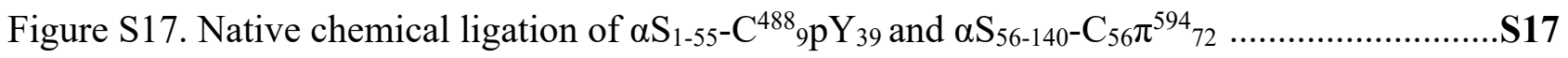

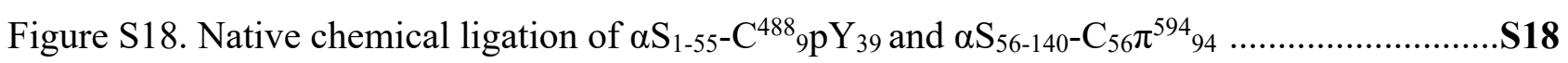

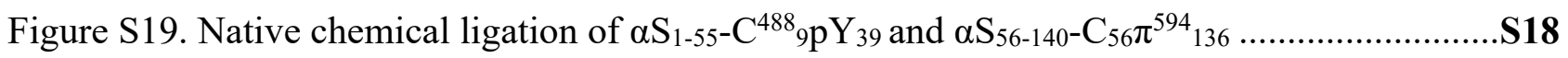

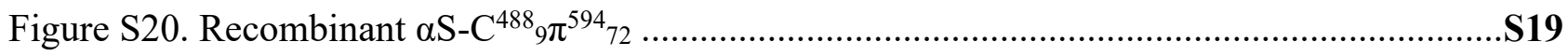

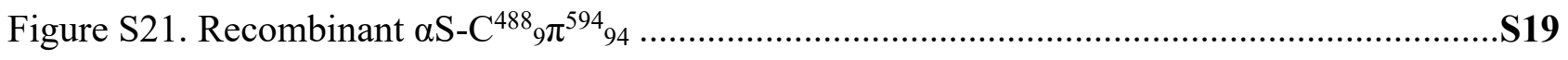

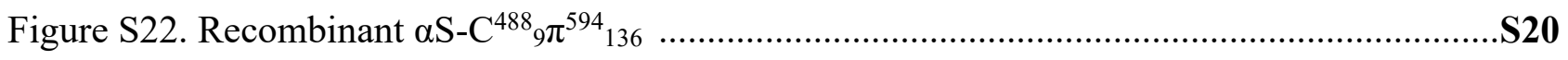

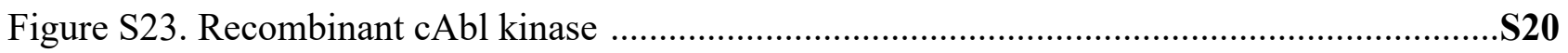

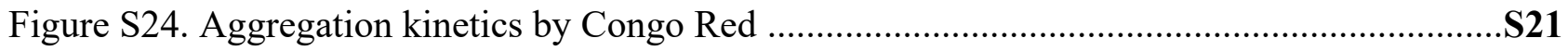

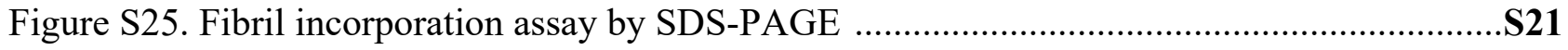

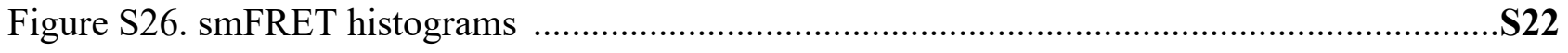

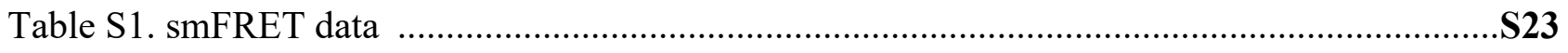

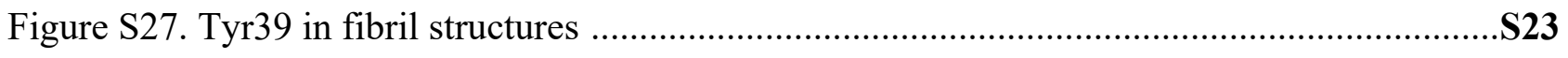

Figure S28. Transmission Electron Microscopy images of $\mathrm{pY}_{39}$ fibrils .....................................S24

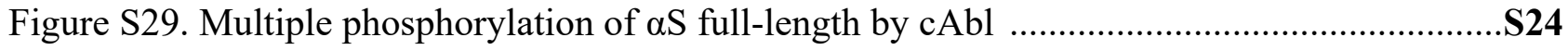




\section{General Information}

Reagents for peptide synthesis, including2-(1H-benzotriazol-1-yl)-1,1,3,3tetramethyluronium hexafluorophosphate (HBTU), N,N-Diisopropylethylamine (DIPEA), and Fluorenylmethyloxycarbonyl (Fmoc) amino acids, were purchased from EMD Millipore (Burlington, MA). Reagents for NCL, including $\mathrm{NaNO}_{2}$, Tris(2-carboxyethyl)phosphine (TCEP), and mercaptophenylacetic acid (MPAA), were purchased from Sigma-Aldrich (St. Louis, MO). Tyrosine starting material was purchased from Spectrum Chemical (New Brunswick, NJ) for the synthesis of propargyltyrosine. NMR spectra were obtained on a Bruker DRX 500 spectrometer (Bruker; Billerica, MA). Alexa Fluor 488 (AF488) and 594 (AF594) were purchased from Thermo Fisher Scientific (Waltham, MA). E. coli BL21(DE3) cells were purchased from Stratagene (La Jolla, CA). DNA oligomers were purchased from Integrated DNA Technologies, Inc (Coralville, IA). Buffers were made with MilliQ filtered (18 M $\Omega$ ) water (Millipore; Billerica, MA). Preparation of the pTXB1$\alpha \mathrm{S}$-intein- $\mathrm{H}_{6}$ plasmid containing $\alpha \mathrm{S}$ with a C-terminal fusion to the Mycobacterium xenopi GyrA intein and C-terminal His 6 tag was described previously. ${ }^{l}$ This plasmid was used as a starting point for the preparation of $\alpha \mathrm{S}$ fragment-intein constructs. Matrix-assisted laser desorption/ionization (MALDI) mass spectra were collected with a Bruker Ultraflex III MALDI-TOF/TOF mass spectrometer (Billerica, MA). UV/vis absorbance spectra were obtained with a Hewlett-Packard 8452A diode array spectrophotometer (currently Agilent Technologies). Gel images were obtained with a Typhoon FLA 7000 (GE Lifesciences; Princeton, NJ). Congo Red absorbance spectra were collected on a Tecan M1000 plate reader (Mannedorf, Switzerland). Peptides and protein fragments were purified on a Varian preparative HPLC equipped with fraction collector and diode array detector (Agilent Technologies; Santa Clara, CA). Transmission electron microscopy (TEM) images were collected on a FEI Tecnai 12 electron microscope (Hillsboro, OR). 


\section{Production of recombinant $\alpha S$ constructs}

A plasmid containing an $\alpha \mathrm{S}$ construct fused to a polyhistidine-tagged GyrA intein from Mycobacterium xenopi (Mxe) was transformed into BL21 DE3 competent cells by heat shocking at $42{ }^{\circ} \mathrm{C} .{ }^{2}$ Cells were grown on Ampicillin (Amp) plates, and single colonies were picked to inoculate primary cultures in LB media supplemented with $1 \mu \mathrm{g} / \mathrm{mL}$ Amp. Secondary cultures were incubated at $37{ }^{\circ} \mathrm{C}$ in a shaker at $250 \mathrm{rpm}$ until optical density (OD) reached $\sim 0.6$. Expression of the gene of interest was induced with isopropyl $\beta$-D-1-thiogalactopyranoside (IPTG). Cells were then grown in the shaker-incubator at $18{ }^{\circ} \mathrm{C}$ overnight. After centrifugation $\left(5000 \mathrm{rpm}, 20 \mathrm{~min}, 4^{\circ} \mathrm{C}\right)$, cell pellets were re-suspended in buffer (20 mM Tris, $5 \mathrm{mM}$ ethylenediaminetetraacetic acid (EDTA), 1 Roche protease inhibitor tablet) and sonicated in a cup in an ice bath ( $5 \mathrm{~min}, 1 \mathrm{~s} \mathrm{ON}, 1 \mathrm{~s} \mathrm{OFF})$. The resulting lysate was centrifuged $\left(14,000 \mathrm{rpm}, 25 \mathrm{~min}, 4^{\circ} \mathrm{C}\right)$, and supernatant containing the protein of interest (POI) was purified over a Ni-NTA affinity column. For C-terminal carboxylate constructs, intein cleavage was carried out by incubation with $200 \mathrm{mM} \beta$-mercaptoethanol ( $\beta \mathrm{ME}$ ) on a rotisserie over night at room temperature. For C-terminal thioester constructs, excess imidazole was removed by dialysis before transthioesterification with $200 \mathrm{mM}$ sodium 2-mercaptoethanesulfonate (MESNa) was carried out overnight at $4{ }^{\circ} \mathrm{C}$ on a stir plate. Cleaved POI was dialyzed into $20 \mathrm{mM}$ Tris, $\mathrm{pH} 8$ buffer before purification over a second Ni-NTA column to remove the free intein from the sample. Fulllength $\alpha \mathrm{S}$ and C-terminal fragment constructs were purified by fast-protein liquid chromatography (FPLC) using a Hi-Trap Q $5 \mathrm{~mL}$ column prior to labeling with fluorophore. Labeling with Alexa Fluor 488 (AF488) maleimide was done by incubation of the POI with tris(2-carboxyethyl)phosphine (TCEP) followed by addition of 2 equiv of fluorophore dissolved in DMSO. The reaction tube was wrapped in aluminum foil and incubated at $37^{\circ} \mathrm{C}$ for $1-3 \mathrm{~h}$. Completion of labeling was verified by matrix-assisted laser desorption ionization mass spectrometry (MALDI-MS). Labeled proteins were purified by reverse-phase high-performance liquid chromatography (RP-HPLC) using a C4 column.

Unnatural amino acid incorporation via amber codon suppression was used to produce doublelabeled full-length $\alpha \mathrm{S}$ and C-terminal fragments bearing Alexa Fluor 594 (AF594). Plasmid containing the desired $\alpha \mathrm{S}$ construct was transformed into E. coli pDULE-pXF cells with pretransformed plasmids encoding propargyl-tyrosine (Ppy or $\pi$ ) synthetase and tRNA. ${ }^{l}$ Expression was carried out as above, except cells were grown in M9 minimal media, and $\pi(220 \mathrm{mg} / \mathrm{L})$ was added to the culture at OD $\sim 0.8$ with a 10 -min incubation prior to inducing expression with IPTG. For fulllength constructs bearing two fluorophores, purified protein was first labeled with AF488-maleimide 
as described above. Upon completion of the first labeling, product was dialyzed into $20 \mathrm{mM}$ Tris $\mathrm{pH}$ 8 and labeled with AF594-azide via copper-catalyzed azide-alkyne cyclization. Catalytic mixture consisting of 2 equiv $\mathrm{CuSO}_{4}, 10$ equiv THPTA, and 20 equiv sodium ascorbate was let sit for $10 \mathrm{~min}$ and added to the protein along with 2 equiv fluorophore. Labeled proteins were purified by HPLC over a $\mathrm{C} 4$ preparatory column.

Deletion PCR was performed on plasmids containing full-length $\alpha \mathrm{S}$ to generate protein fragments used for NCL. Protein fragment constructs were expressed and purified following the same procedures used for full-length protein. For N-terminal protein fragments, expression in $E$ coli results in its production with the initiator methionine, which is cleaved in vivo by endogenous methionine amino peptidase. ${ }^{3}$ The exposed N-terminal cysteine reacts with endogenous aldehydes to form thiazolidine adducts (Thz). ${ }^{4}$ Therefore, de-protection of the thiazolidine ${ }^{5}$ was carried out by reacting it with $100 \mathrm{mM}$ methoxyamine at $\mathrm{pH} 4$ to regenerate the $\mathrm{N}$-terminal cysteine for later use in ligation.

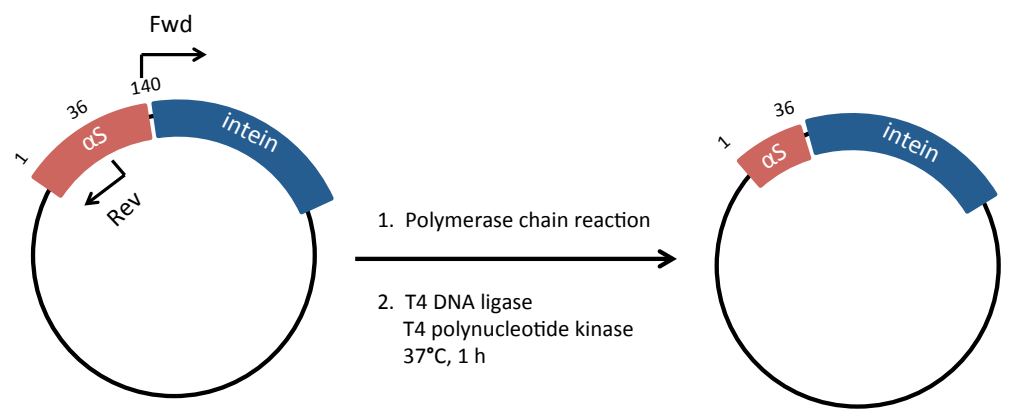

Primer sequences:

\begin{tabular}{|l|l|l|}
\hline$\alpha \mathrm{S}_{1-36}$ & Forward & 5'-TGCATCACGGGAGATGCA-3' \\
\hline & Reverse & 5'-ACCCTCTTTTGTCTTTCCTGC-3' \\
\hline$\alpha \mathrm{S}_{1-55}$ & Forward & 5'-TGCATCACGGGAGATGCA-3' \\
\hline & Reverse & 5'-CACTGTTGCCACACCATG-3' \\
\hline$\alpha \mathrm{S}_{56-140}$ & Forward & 5'-TGTGAGAAGACCAAAGAGC-3' \\
\hline & Reverse & 5'-CATATGTATATCTCCTTCTTAAAGTTAAAC-3' \\
\hline$\alpha{\mathrm{S}-\mathrm{E}_{39}}$ & Forward & 5'-GGAAAGACAAAAGAGGGTGTTCTCGAAGTAGGCTCC-3' \\
\hline & Reverse & 5'-GGAGCCTACTTCGAGAACACCCTCTTTTGTCTTTCC-3' \\
\hline
\end{tabular}


Solid phase peptide synthesis (SPPS) of peptides

Peptides needed for the semi-synthesis of $\alpha \mathrm{S}-\mathrm{pY}_{39}$ were made via solid phase peptide synthesis (SPPS) on 2-chloro-trityl resin following standard procedures. ${ }^{6}$ The resin was derivatized by coupling Fmoc-hydrazine, and extra sites were capped with methanol. For amino acid coupling, 5 equiv Fmoc-protected amino acid were activated with 10 equiv DIPEA and added along with 5 equiv HBTU to the deprotected peptidyl resin with stirring for $30 \mathrm{~min}$. Washes with DMF and DCM were performed between each coupling. Fmoc deprotection was done by stirring for $20 \mathrm{~min}$ with $20 \%$ (v/v) piperidine in DMF. Peptide cleavage from the resin was performed by adding $20 \mu \mathrm{L}$ of cleavage cocktail (95\% TFA, 2.5\% TIPS, 2.5\% H2O) per mg of resin and agitating for $1.5 \mathrm{~h}$ at room temperature. The peptidyl hydrazides $\alpha \mathrm{S}_{30-55}-\mathrm{C}_{30} \mathrm{pY}_{39}-\mathrm{NHNH}_{2}$ and $\alpha \mathrm{S}_{37-55}-\mathrm{V} *{ }_{37} \mathrm{p} \mathrm{P}_{39}-\mathrm{NHNH}_{2}$ were synthesized using phosphotyrosine (Fmoc-Tyr $(\mathrm{PO}(\mathrm{OBzl}) \mathrm{OH})-\mathrm{OH})$ at position 39.

To label $\alpha \mathrm{S}_{1-29}-\mathrm{C}_{9}$, the lyophilized peptide was re-dissolved in $20 \mathrm{mM}$ Tris $\mathrm{pH} 8$ and reacted with 2 equiv AF488-maleimide at $37^{\circ} \mathrm{C}$ for $1-4 \mathrm{~h}$ until product formation was observed by MALDIMS. All peptides were purified by RP-HPLC using a C18 preparatory column.

\section{c-Abl kinase expression and purification}

The plasmids ABL1_HUMAN_D0 and YopH were gifts from John Chodera, Nicholas Levinson, and Markus Seeliger (Addgene plasmid \#79727; http://n2t.net/addgene:79727; RRID: Addgene_79727). (Addgene plasmid \#79749; http://n2t.net/addgene:79749; RRID: Addgene_79749). ${ }^{7} \mathrm{c}-\mathrm{Abl}$ and YopH were co-transformed into BL21 cells and grown on plates overnight. Cultures were grown to $\mathrm{OD}_{600}$ of 0.6 , and expression was induced with $0.25 \mathrm{mM}$ IPTG. The induced culture was grown with shaking at $16{ }^{\circ} \mathrm{C}$ overnight. Cells were resuspended in $40 \mathrm{mM}$ Tris, pH 8.3 buffer with 1 protease inhibitor tablet and 5\% glycerol. Buffers for the Ni-NTA column were also supplemented with $5 \%$ glycerol. TEV protease was added to eluate from Ni-NTA purification, and the sample was dialyzed into $20 \mathrm{mM}$ Tris $\mathrm{pH} 8.0$ at $4{ }^{\circ} \mathrm{C}$ overnight. The c-Abl was purified by FPLC over a HiTrap Q column using buffers containing $1 \mathrm{mM}$ dithiothreitol (DTT) and $5 \%$ glycerol, followed by size exclusion chromatography over a Superdex 75 column. Pooled pure fractions of c-Abl were aliquotted and stored at $-80^{\circ} \mathrm{C}$ in buffer consisting of $20 \mathrm{mM}$ Tris $\mathrm{pH} 8.0,100$ $\mathrm{mM} \mathrm{NaCl}, 1 \mathrm{mM}$ DTT, 5\% glycerol. 
In vitro phosphorylation of full-length $\alpha S$ and $\alpha S$ fragments

WT $\alpha \mathrm{S}$, or $\alpha \mathrm{S}_{1-55}$, or $\alpha \mathrm{S}_{1-55-} \mathrm{C}_{9}{ }^{488}$ fragment was dissolved in buffer (50 mM Tris, $150 \mathrm{mM}$ $\mathrm{NaCl}, \mathrm{pH}$ 7.4) to a final concentration of $\sim 70 \mu \mathrm{M}$. To the sample was added up to 0.1 equiv c-Abl enzyme, Mg-ATP to a final concentration of $2 \mathrm{mM}$, and $\mathrm{MgCl}_{2}$ to a final concentration of $5 \mathrm{mM}$. The reaction was incubated in a $30{ }^{\circ} \mathrm{C}$ water bath for several $\mathrm{h}$. The reaction was monitored by MALDIMS and supplemented with additional Mg-ATP and $\mathrm{MgCl}_{2}$ as necessary. Phosphorylated $\alpha \mathrm{S}_{1-55}$ or $\alpha \mathrm{S}_{1-55}-\mathrm{C}_{9} 488$ fragment was purified by RP-HPLC over a C18 column.

\section{Native chemical ligation (NCL)}

Peptide-acyl-hydrazide was dissolved in low pH NCL buffer (6 M GdnHCl, $200 \mathrm{mM} \mathrm{Na}_{2} \mathrm{PO}_{4}$, $\mathrm{pH} 3$ ) for a final concentration of $2 \mathrm{mM}$ and chilled to $-15^{\circ} \mathrm{C}$ in an ice-salt bath. Hydrazide to azide conversion ${ }^{8}$ was achieved by adding 10 equiv $\mathrm{NaNO}_{2}$ and agitating by magnetic stirring for $15 \mathrm{~min}$ at $-15^{\circ} \mathrm{C}$. The partner peptide, pre-dissolved in NCL buffer $\mathrm{pH} 7.0$ along with 40 equiv MPAA, was added to the reaction. The mixture was warmed to room temperature, and the $\mathrm{pH}$ was adjusted to 7.0. Reaction was incubated at $37^{\circ} \mathrm{C}$ with agitation and supplemented with TCEP as necessary. Product formation was monitored by MALDI-MS.

For ligation of expressed N-terminal protein fragment, $\alpha \mathrm{S}$ fragment-MxeHis6 was purified by Ni-NTA, and intein was cleaved with $200 \mathrm{mM}$ MESNa to generate a thioester. ${ }^{9}$ The protein fragmentthioester was purified by RP-HPLC over a C18 column and lyophilized. To carry out ligation, the partner protein fragment containing a ligation handle was re-dissolved in NCL buffer (6 M GdnHCl, $200 \mathrm{mM} \mathrm{Na} 2 \mathrm{PO}_{4}, 30 \mathrm{mM}$ TCEP, $50 \mathrm{mM}$ MPAA, $\mathrm{pH}$ 7.0). The protein fragment-thioester was added, and reaction was incubated at $37^{\circ} \mathrm{C}$ with agitation at $250 \mathrm{rpm}$ for several hours to overnight. Reaction was monitored by MALDI-MS, and the product was reduced with $300 \mathrm{mM}$ TCEP and purified by RP-HPLC. To convert cysteines and penicillamines used in ligation to the respective native alanines and valines, the protein was incubated with $50 \mathrm{mM}$ radical initiator VA-044 and $10 \%(\mathrm{v} / \mathrm{v}) t-\mathrm{BuSH}$ in an argon-purged tube at $37^{\circ} \mathrm{C}$ overnight. The full-length $\alpha \mathrm{S}$ products were then purified by RPHPLC. 


\section{Protein aggregation kinetics and percentage incorporation into fibrils}

Protein samples (100 $\mu \mathrm{M}$ total concentration in monomer units) in buffer (20 mM Tris, 100 $\mathrm{mM} \mathrm{NaCl}, \mathrm{pH}$ 7.5) were prepared in triplicate in Eppendorf tubes. Samples were shaken in an Ika MS3 orbital shaker set to $1300 \mathrm{rpm}$ at $37^{\circ} \mathrm{C}$. At each time point, a $10 \mu \mathrm{L}$ aliquot from each sample was added to $140 \mu \mathrm{L}$ of Congo Red solution ( $20 \mu \mathrm{M}$ in $20 \mathrm{mM}$ Tris, $100 \mathrm{mM} \mathrm{NaCl}, \mathrm{pH} 7.5)$ in a clean Eppendorf tube and incubated for 10-15 min. Samples were transferred to 96-well clear bottom CoStar plates. Absorbance was measured on a Tecan M1000 using 230-700 nm range, $1 \mathrm{~nm}$ step size, 25 flashes/read. The extent of aggregation was determined based on the ratio of Congo Red absorbance at $540 \mathrm{~nm} / 480 \mathrm{~nm}$. After the final time point, samples were spun down at maximum speed on a tabletop centrifuge for $90 \mathrm{~min}$. The supernatant was removed, and pellet was resuspended in the original volume of buffer. Samples were supplemented with SDS to $25 \mathrm{mM}$ final concentration, boiled for $20 \mathrm{~min}$, and chilled on ice. Monomeric samples for calibration were prepared by 2 -fold serial dilutions in water. All samples were analyzed by SDS-PAGE (18\% acrylamide, $150 \mathrm{~V}, 1.5 \mathrm{~h}$ ). Gels were stained with Coomassie Brilliant Blue dye. Quantification of the intensity of bands was done using the ImageJ software.

\section{Single molecule Förster resonance energy transfer (smFRET)}

All smFRET measurements were made on a MicroTime 200 inverse time- resolved confocal microscope (PicoQuant, Berlin, Germany). Eight-chambered Nunc coverslips (Thermo Fisher Scientific, Waltham, MA) were plasma cleaned and coated with poly(ethylene glycol) poly(L-lysine) overnight. For each smFRET measurement, buffer (20 mM Tris, $100 \mathrm{mM} \mathrm{NaCl}, \mathrm{pH}$ 7.4) was added to a chamber, followed by $30 \mathrm{pM} \alpha \mathrm{S}$ labeled with AF488 and AF594. Samples were mixed by pipetting and incubated for 5 min before measurement. $485 \mathrm{~nm}$ and $560 \mathrm{~nm}$ lasers pulsed at $40 \mathrm{MHz}$ were adjusted to $30 \mu \mathrm{W}$ before entering the microscope. Fluorescence was collected through the objective and passed through a $100 \mu \mathrm{m}$ pinhole. Excitation and emission were discriminated by passing the photons through a HQ585LP dichroic in combination with ET525/50M and HQ600LP filters. Signal was detected by photodiodes. Photon traces were collected in 1-ms time bins for an hour. A threshold of 30 counts $/ \mathrm{ms}$ total in the donor and acceptor channels was used to discriminate events from noise. For each event, the energy transfer efficiency between donor and acceptor fluorophore $\left(\mathrm{ET}_{\text {eff }}\right)$ was calculated in the SymPhoTime 64 software using the following equation: 


$$
\mathrm{ET}_{\text {eff }}=\frac{\mathrm{I}_{\mathrm{a}}-\beta \mathrm{I}_{\mathrm{d}}}{\left(\mathrm{I}_{\mathrm{a}}-\beta \mathrm{I}_{\mathrm{d}}\right)+\gamma\left(\mathrm{I}_{\mathrm{d}}+\beta \mathrm{I}_{\mathrm{d}}\right)}
$$

where $I_{a}$ and $I_{b}$ are respectively the intensity of fluorescence detected in the acceptor and donor channels. $\beta$ is the leakage of the fluorescence from the donor fluorophore into the acceptor channel, measured before each set of experiments. $\gamma$ is the differences in detection efficiency and quantum yield between acceptor and donor fluorophores and is measured every few months. The resulting histograms were fit using Origin (OriginLab Corp, Northampton, MA) to Gaussian distributions:

$$
y=\frac{A}{w \sqrt{\frac{\pi}{2}}} e^{-2\left(\left(\frac{\left(x-x_{c}\right)}{w}\right)^{2}\right)}
$$

where $\mathrm{w}$ is the width, $\mathrm{A}$ is the area, and $\mathrm{x}_{\mathrm{c}}$ is the center of the distribution.

Transmission electron microscopy (TEM)

TEM was carried out on an FEI Tecnai T12 instrument with an accelerating voltage of 120 $\mathrm{kV}$. Fibril samples obtained from aggregation and centrifugation and stored at $-80{ }^{\circ} \mathrm{C}$ as dry pellets were resuspended in $20 \mathrm{mM}$ Tris, $100 \mathrm{mM} \mathrm{NaCl} \mathrm{pH} \mathrm{7.5.} \mathrm{A} 3 \mu \mathrm{L}$ drop of sample was deposited on glow discharged carbon Formvar coated 300-mesh $\mathrm{Cu}$ grids and allowed to rest for 1 minute at room temperature. $3 \mu \mathrm{L}$ of stain ( $2 \% \mathrm{w} / \mathrm{v}$ ammonium molybdate, $\mathrm{pH} 7.8$ in water) was then applied to the grid. The liquid was wicked off with grid paper, and another $3 \mu \mathrm{L}$ of stain was applied and wicked off. Images were collected at magnifications ranging from $6500 \mathrm{x}$ to $42000 \mathrm{x}$. 
(A)

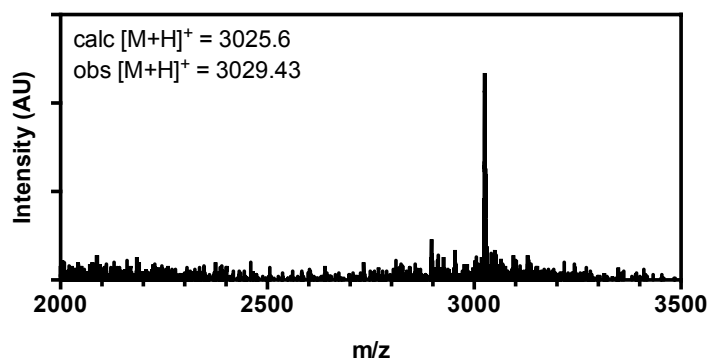

(B)

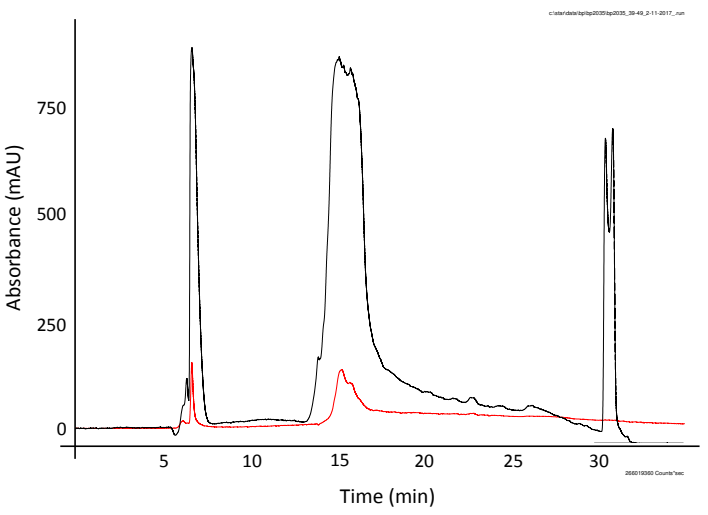

Figure S1. Synthetic peptide $\alpha \mathrm{S}_{1-29-} \mathrm{C}^{488}{ }_{9}$ with C-terminal hydrazide (A) MALDI-MS (B) HPLC

(A)

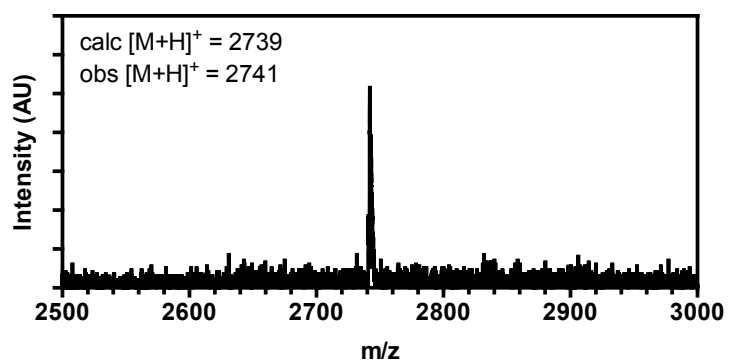

(B)

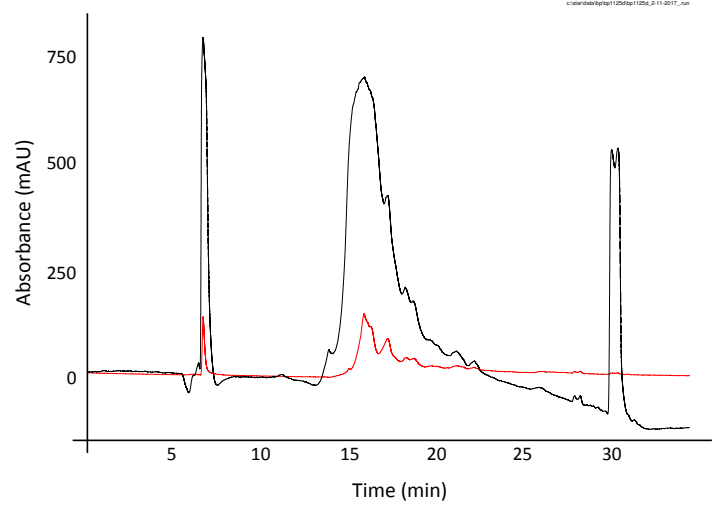

Figure S2. Synthetic peptide $\alpha \mathrm{S}_{30-55}-\mathrm{C}_{30} \mathrm{pY}_{39}$ with C-terminal hydrazide (A) MALDI-MS (B) HPLC 
(A)

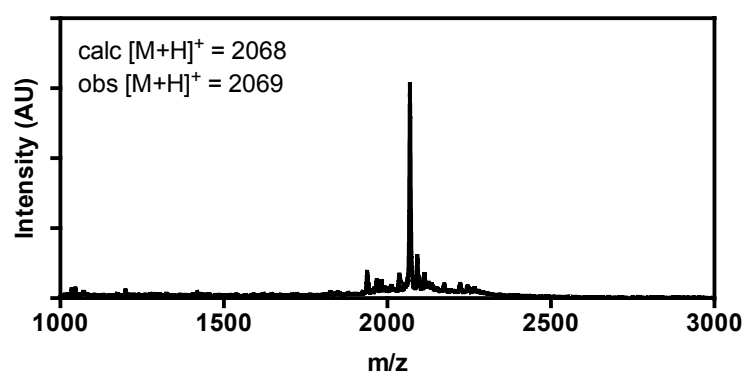

(B)

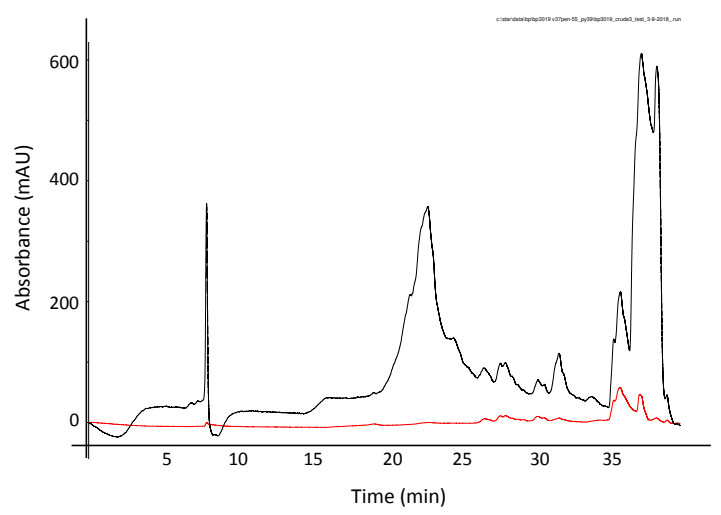

Figure S3. Synthetic peptide $\alpha_{37-55}-\mathrm{V}^{*}{ }_{37} \mathrm{pY}_{39}$ with C-terminal hydrazide (A) MALDI-MS (B) HPLC

(A)

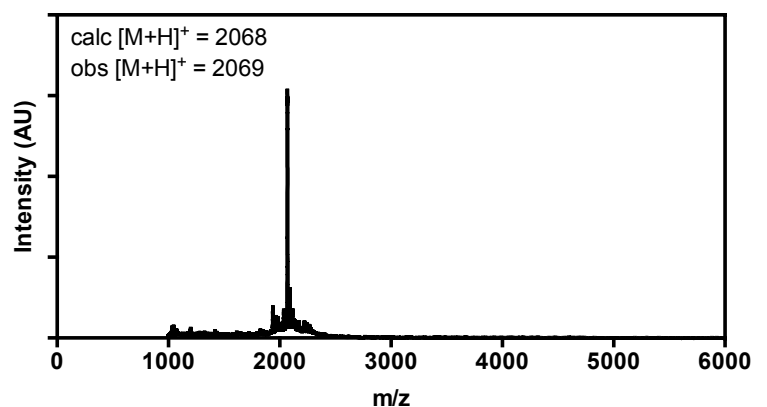

(B)

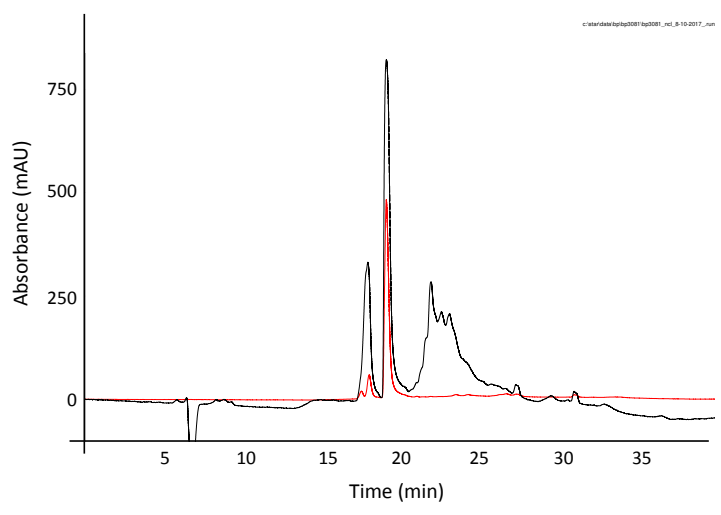

Figure S4. Native chemical ligation of $\alpha \mathrm{S}_{1-29}-\mathrm{C}^{488}$ and $\alpha \mathrm{S}_{30-55}-\mathrm{C}_{30} \mathrm{p} \mathrm{Y}_{39}$ (A) MALDI-MS (B) HPLC 
(B)
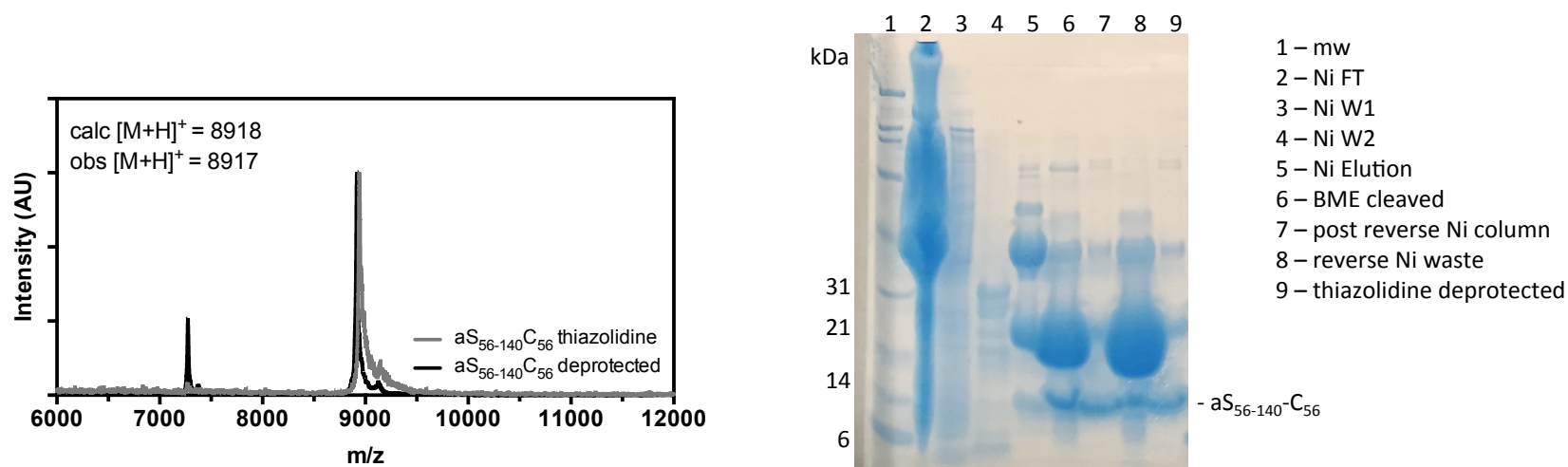

Figure S5. Recombinant $\boldsymbol{\alpha S}_{56-140}-\mathbf{C}_{56}$ (A) MALDI-MS (B) 15\% polyacrylamide SDS-PAGE gel (Coomassie stain)

(A)

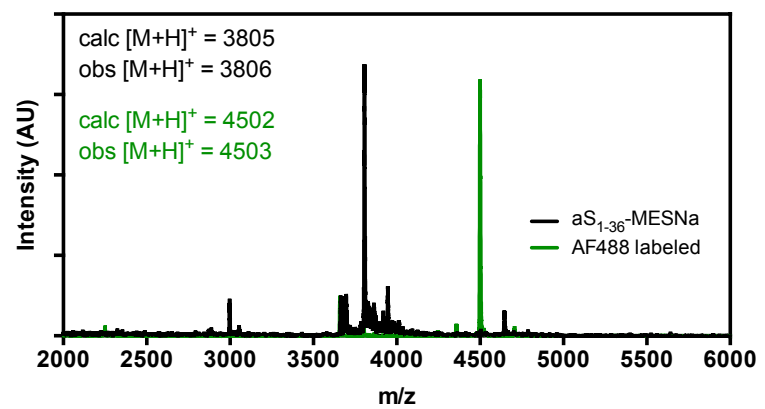

(B)

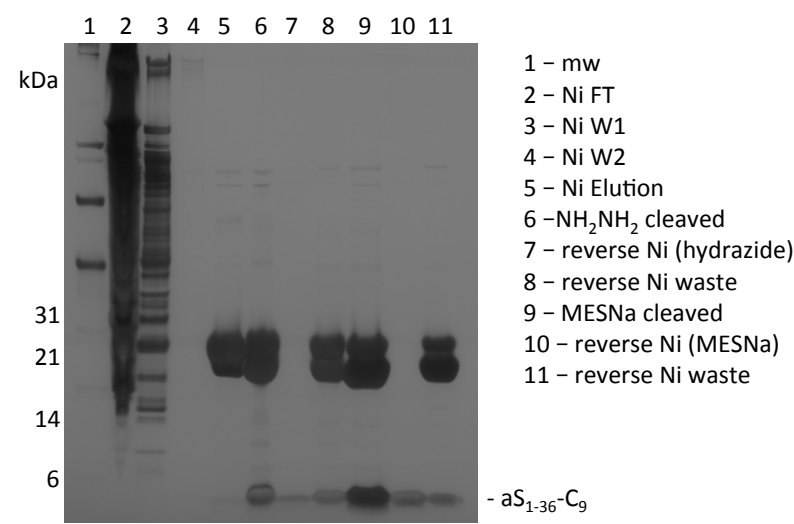

Figure S6. Recombinant $\boldsymbol{\alpha S}_{1-36}-\mathrm{C}_{9}$ and AF488 labeling (A) MALDI-MS (B) $\mathbf{1 5 \%}$ polyacrylamide SDS-PAGE gel (Coomassie stain) 

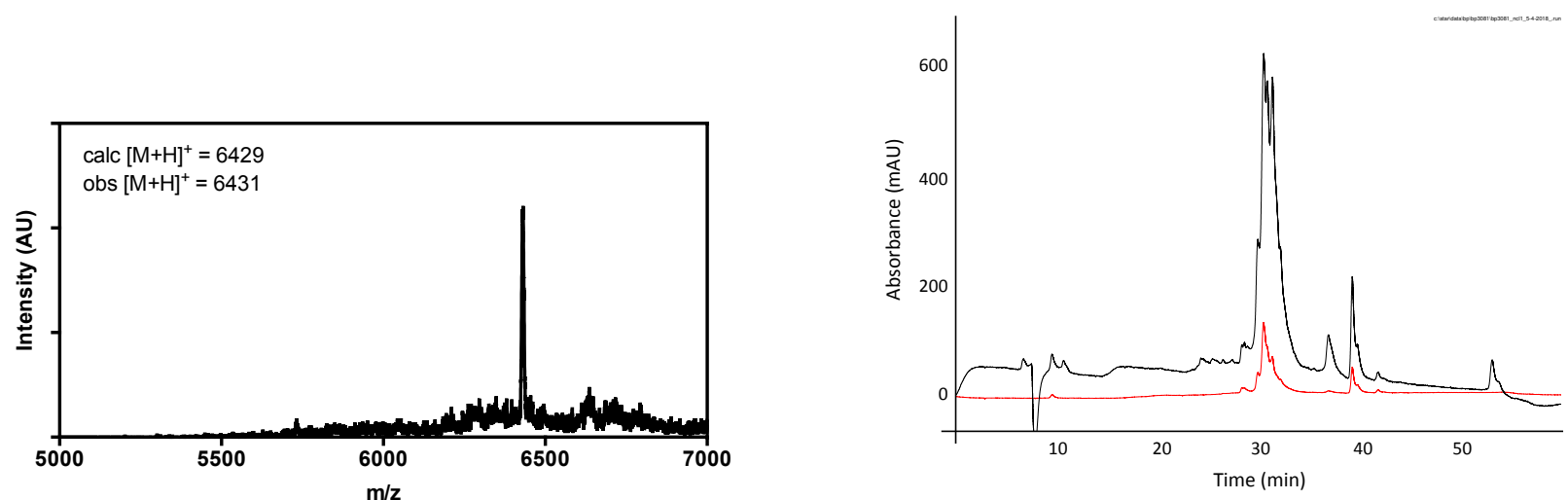

Figure S7. Native chemical ligation of $\alpha_{1-36}-C^{488}$ and $\alpha S_{37-55}-V^{*}{ }_{37} p Y_{39}$ (A) MALDI-MS (B) HPLC

(A)

(B)
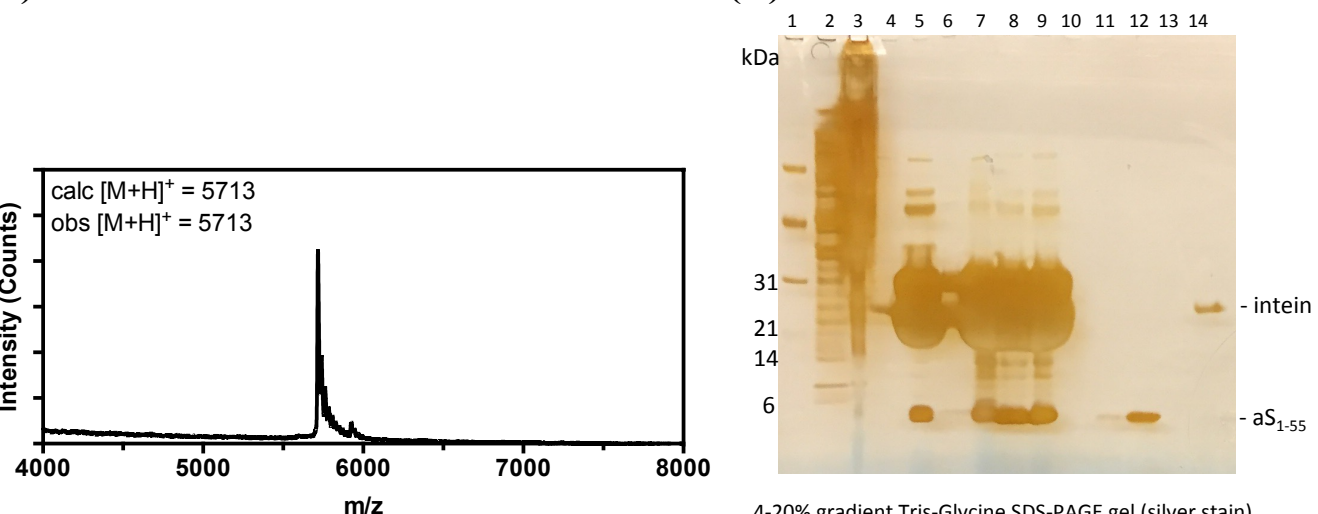

$1-\mathrm{mw}$

2- Ni W1

$3-\mathrm{NiFT}$

4- Ni W2

5 - Elution $(0-6 \mathrm{~mL})$

6 - Elution $(6-12 \mathrm{~mL})$

7 - MESNa cleaved

8 - dialyzed

9 - filtered

10 - SP column FT

11 - FPLC fractions $16-21$

12 - FPLC fractions 22-32

13 - FPLC fractions $42-46$

14 - FPLC waste (free intein)

Figure S8. Recombinant $\boldsymbol{\alpha} \mathbf{S}_{1-55}$ (A) MALDI-MS (B) 15\% polyacrylamide SDS-PAGE gel (Silver stain)

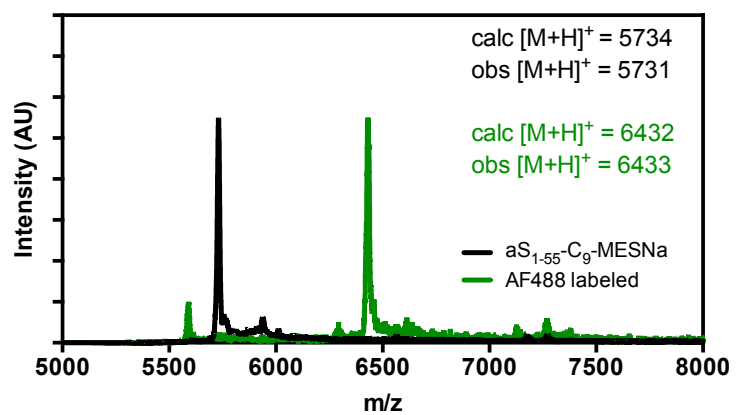

Figure S9. Recombinant $\alpha \mathrm{S}_{1-55}-\mathrm{C}_{9}$ and AF488 labeling MALDI-MS 
(A)

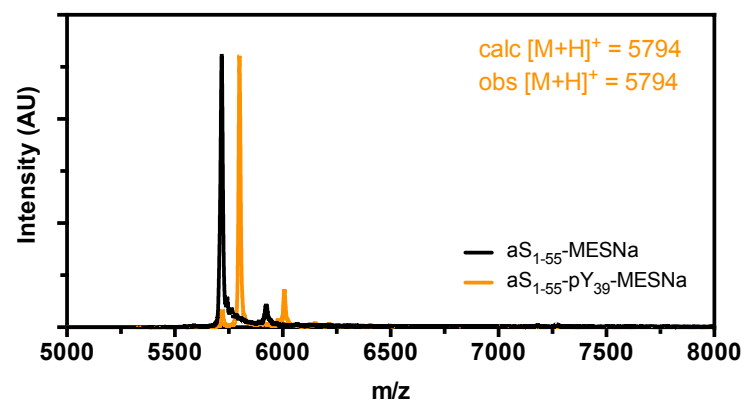

(B)

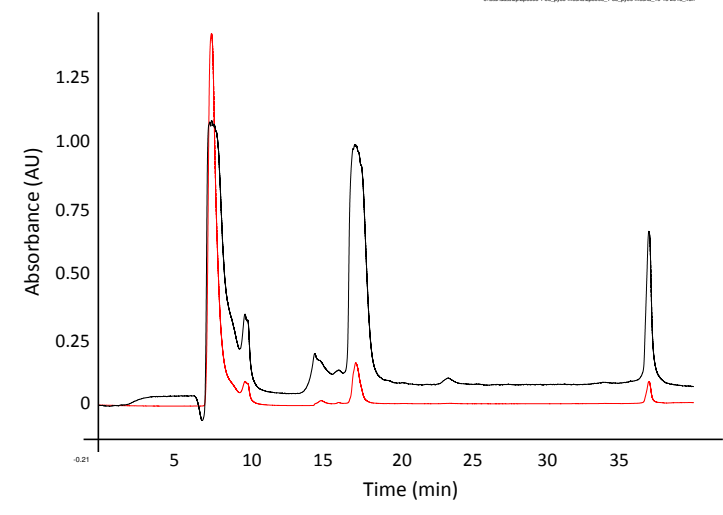

Figure S10. In vitro phosphorylation of $\alpha \mathbf{S}_{1-55}$ by cAbl kinase (A) MALDI-MS (B) HPLC

(A)

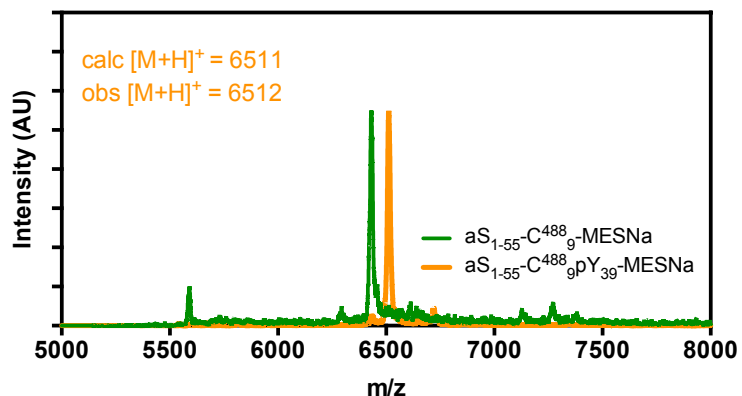

(B)

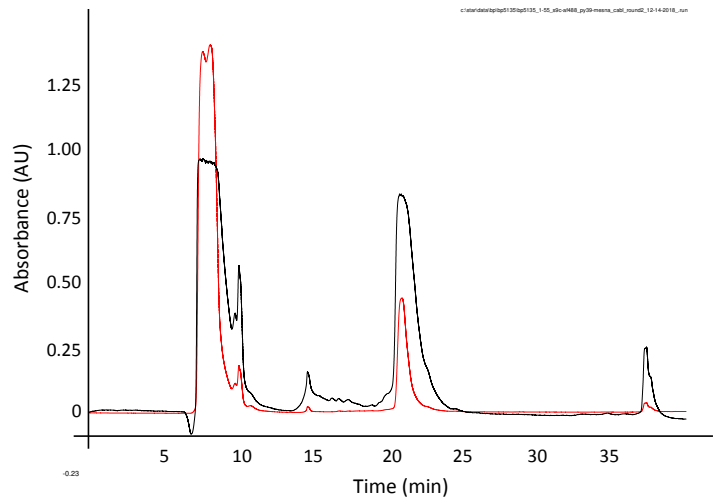

Figure S11. In vitro phosphorylation of $\alpha_{\mathbf{S}_{1-55}-\mathrm{C}^{488}}{ }_{9}$ by cAbl kinase (A) MALDI-MS (B) HPLC 
(A)

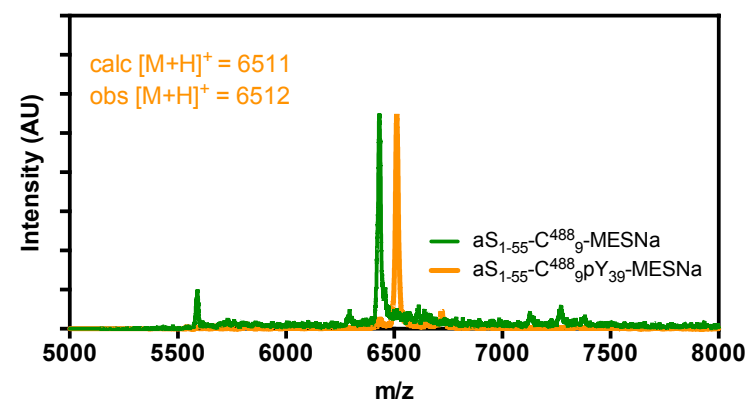

(B)

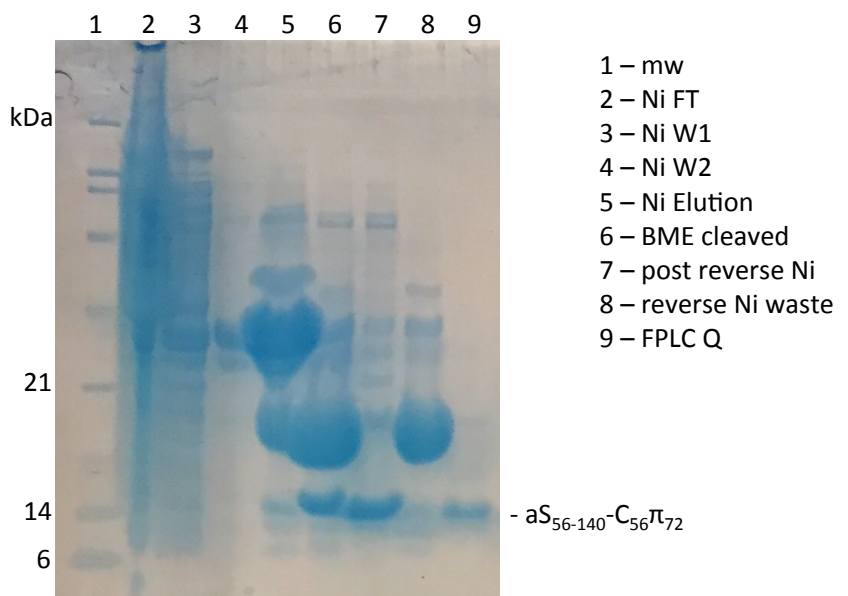

Figure S12. Recombinant $\alpha \mathbf{S}_{56-140}-\mathbf{C}_{56} \pi_{72}$ and AF594 labeling (A) MALDI-MS (B) $15 \%$ polyacrylamide SDS-PAGE gel (Coomassie stain)

(A)

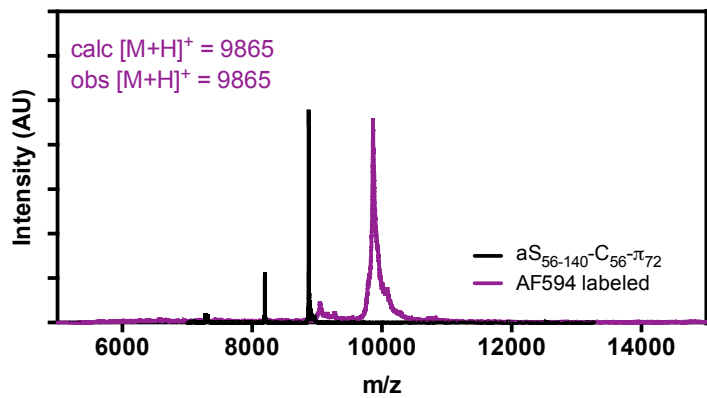

(B)

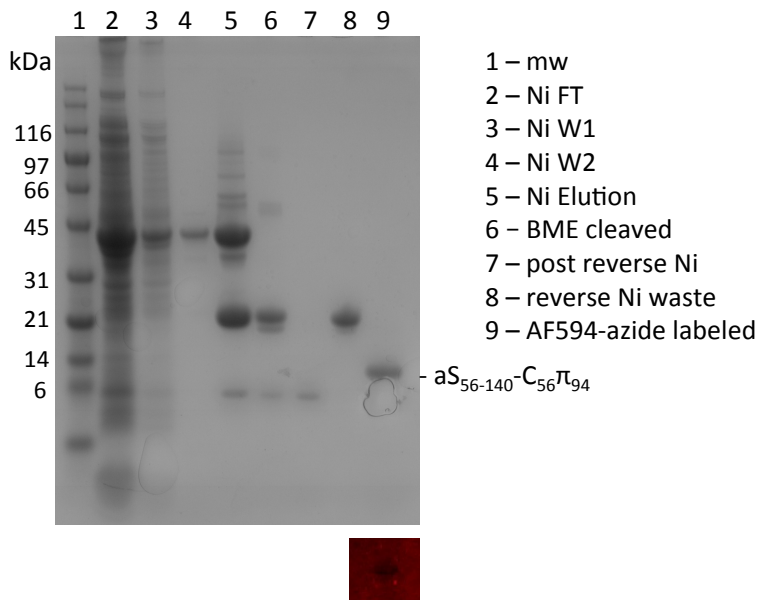

Figure S13. Recombinant $\alpha_{56-140}-\mathbf{C}_{56} \pi_{94}$ and AF594 labeling (A) MALDI-MS (B) $15 \%$ polyacrylamide SDS-PAGE gel (Coomassie stain) and cy5 fluorescence filters 

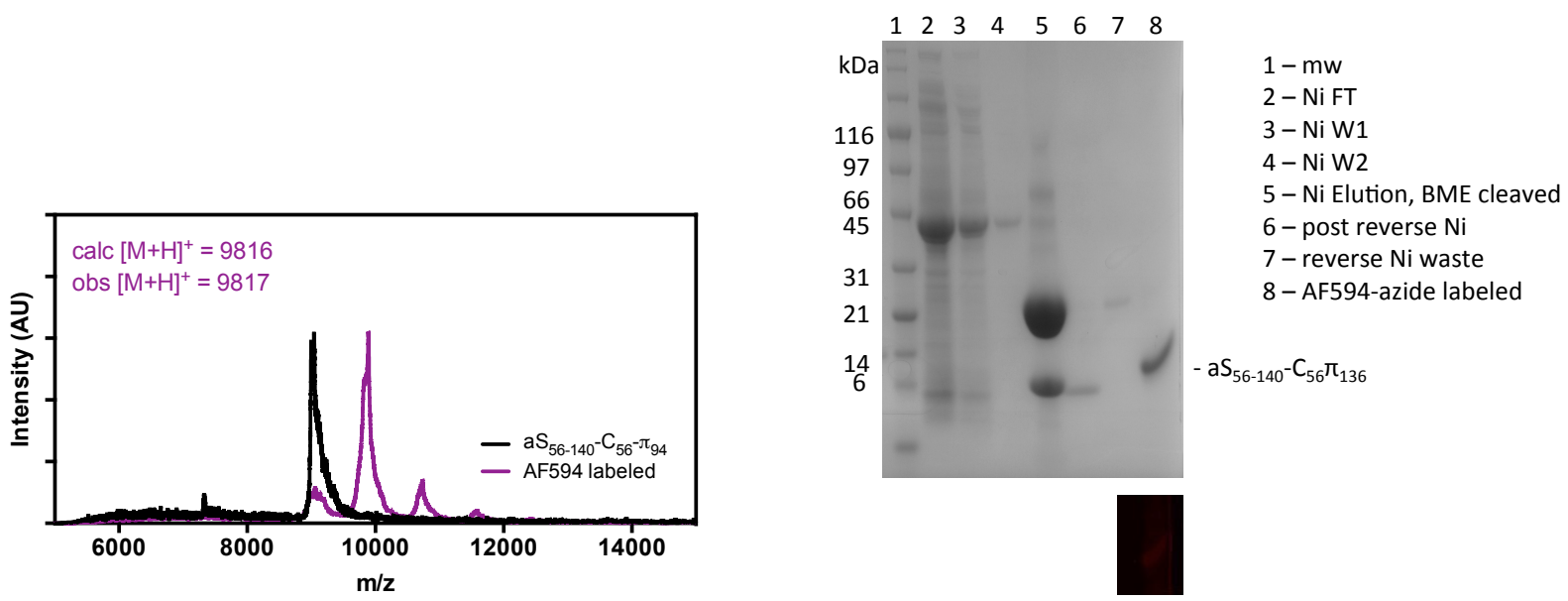

Figure S14. Recombinant $\boldsymbol{\alpha S}_{56-140}-\mathbf{C}_{56} \boldsymbol{\pi}_{136}$ and AF594 labeling (A) MALDI-MS (B) $15 \%$ polyacrylamide SDS-PAGE gel (Coomassie stain) and cy5 fluorescence filters

(A)

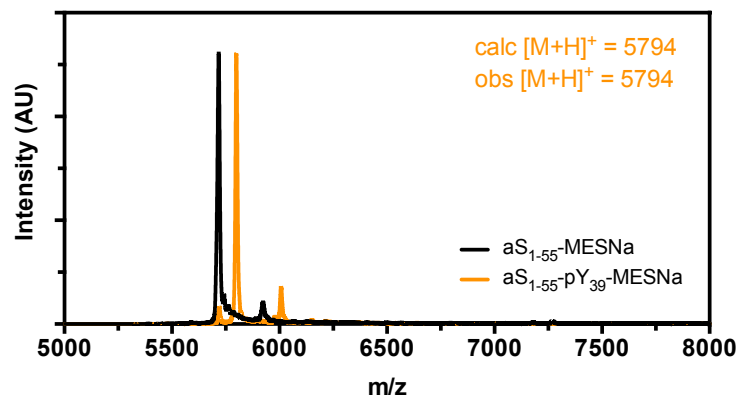

(B)

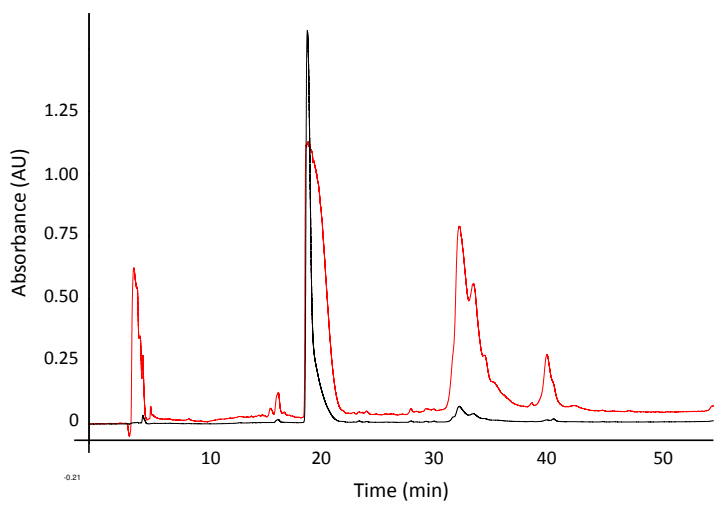

Figure S15. Native chemical ligation of $\alpha \mathrm{S}_{1-55}-\mathrm{p} \mathrm{Y}_{39}$ and $\alpha \mathrm{S}_{56-140}-\mathrm{C}_{56}$ (A) MALDI-MS of ligation product (MPAA adduct) and desulfurized product. (B) HPLC 

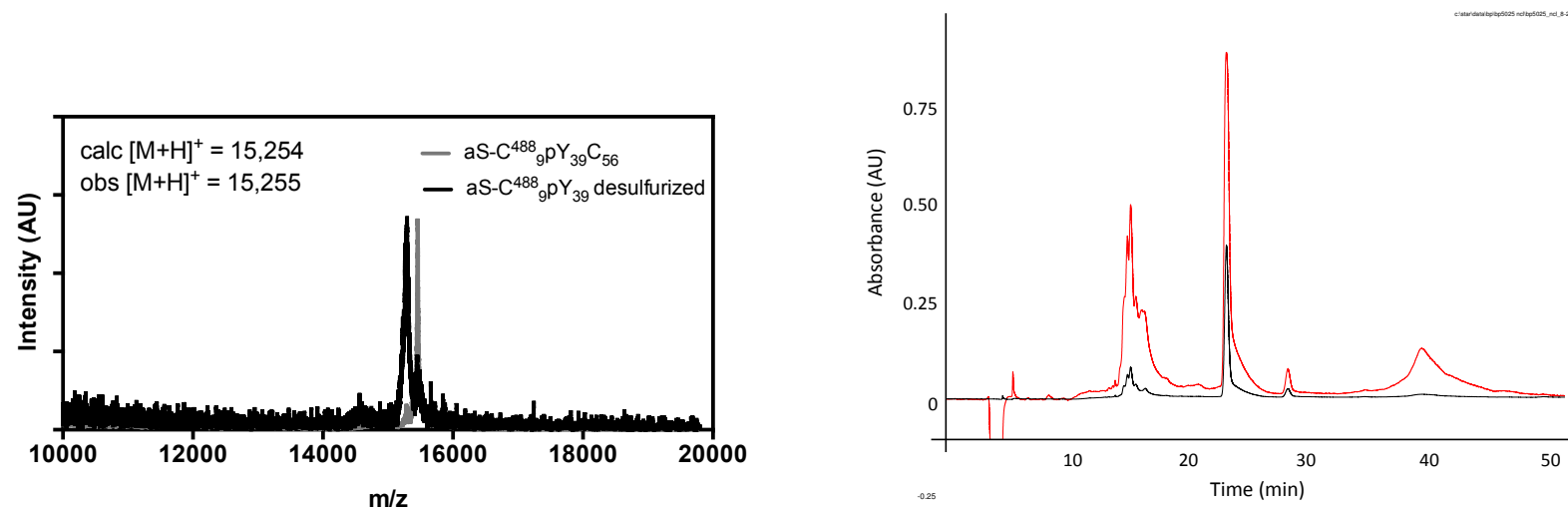

Figure S16. Native chemical ligation of $\alpha \mathrm{S}_{1-55}-\mathrm{C}_{9}^{488} \mathrm{pY}_{39}$ and $\alpha \mathrm{S}_{56-140}-\mathrm{C}_{56}$ (A) MALDI-MS of ligation product (MPAA adduct) and desulfurized product. (B) HPLC

(A)

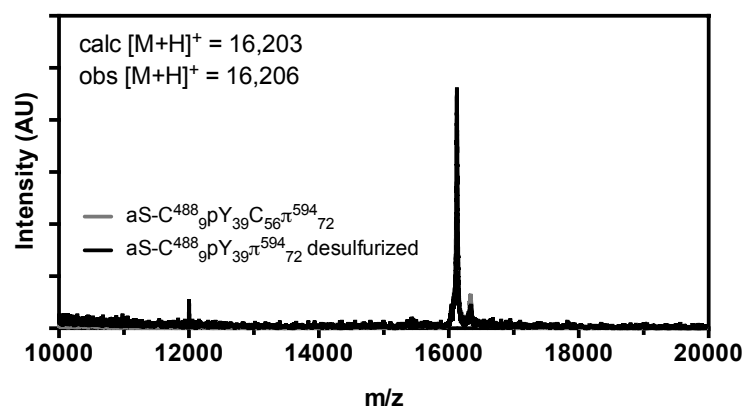

(B)

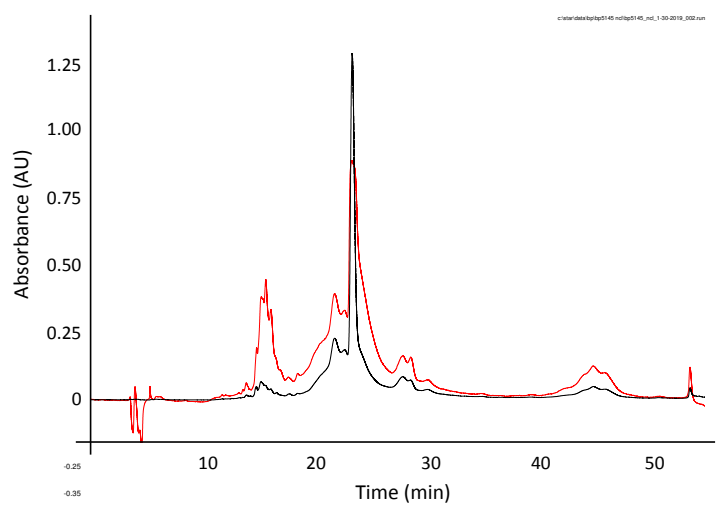

Figure S17. Native chemical ligation of $\alpha \mathrm{S}_{1-55}-\mathrm{C}_{9}{ }^{488} \mathrm{pY}_{39}$ and $\alpha \mathrm{S}_{56-140}-\mathrm{C}_{56} \pi^{594}{ }_{72}$ (A) MALDI-MS (B) HPLC 
(B)
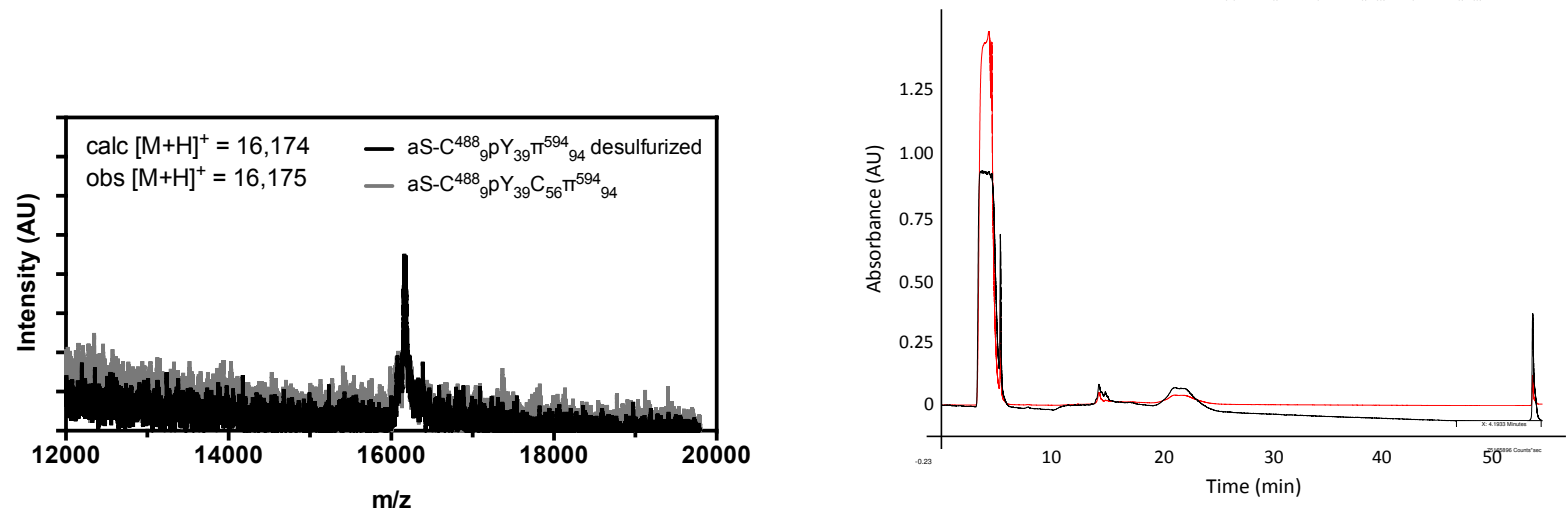

Figure S18. Native chemical ligation of $\alpha S_{1-55}-C_{9}{ }^{488} \mathrm{pY}_{39}$ and $\alpha S_{56-140}-C_{56} \pi^{594}{ }_{94}$ (A) MALDI-MS (B) HPLC

(A)

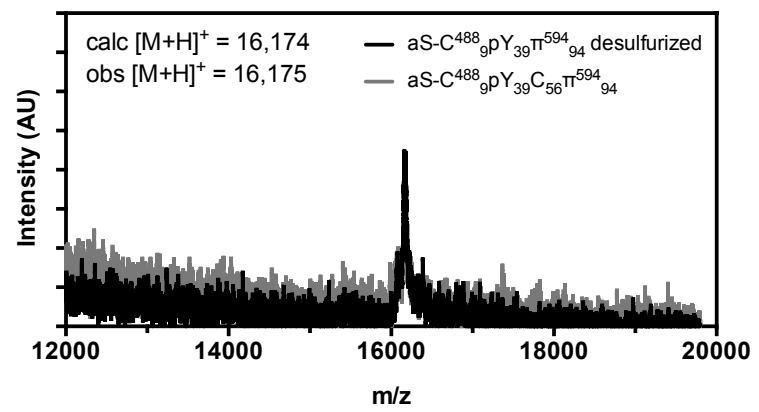

(B)

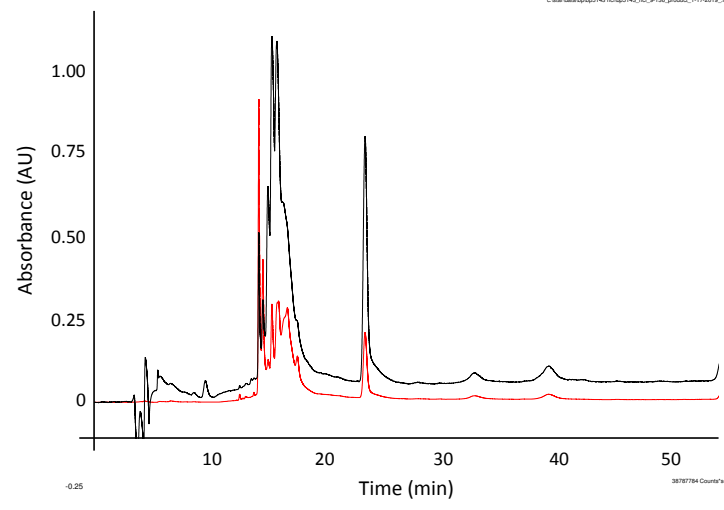

Figure S19. Native chemical ligation of $\alpha \mathrm{S}_{1-55}-\mathrm{C}_{9}{ }^{488} \mathrm{pY}_{39}$ and $\alpha \mathrm{S}_{56-140}-\mathrm{C}_{56} \pi^{594}{ }_{136}$ (A) MALDI-MS (B) HPLC 
(B)
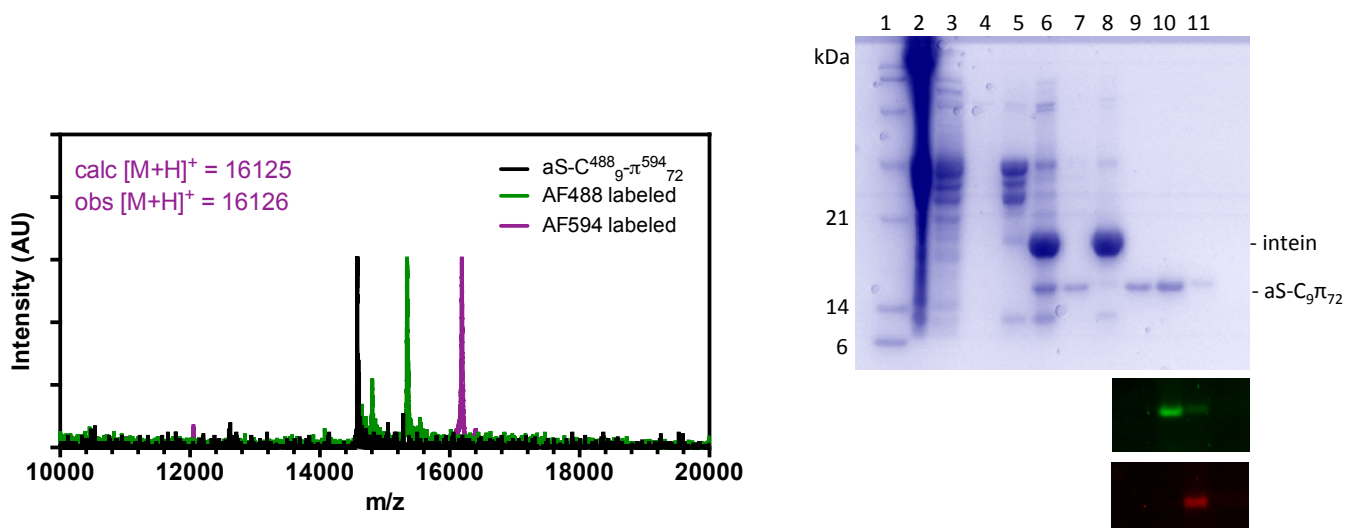

$$
\begin{aligned}
& 1-\mathrm{mW} \\
& 2-\mathrm{Ni} \mathrm{FT} \\
& 3-\mathrm{NiW} 1 \\
& 4-\mathrm{Ni} \text { W2 } \\
& 5-\mathrm{Ni} \text { Elution } \\
& 6-\mathrm{BME} \text { cleaved } \\
& 7-\text { post reverse } \mathrm{Ni} \\
& 8-\text { reverse Ni waste } \\
& 9-\mathrm{AF488-maleimide} \mathrm{labeled} \\
& 10-\mathrm{AF594} \text {-azide labeled } \\
& 11-\mathrm{FPLC} \mathrm{Q}
\end{aligned}
$$

Figure S20. Recombinant $\boldsymbol{\alpha S}-\mathbf{C}_{\boldsymbol{9}^{\mathbf{4 8 8}}} \boldsymbol{\pi}^{\mathbf{5 9 4}}{ }_{72}$ (A) MALDI-MS (B) $15 \%$ polyacrylamide SDS-PAGE gel (Coomassie stain) and FITC and cy5 fluorescence filters

(A)

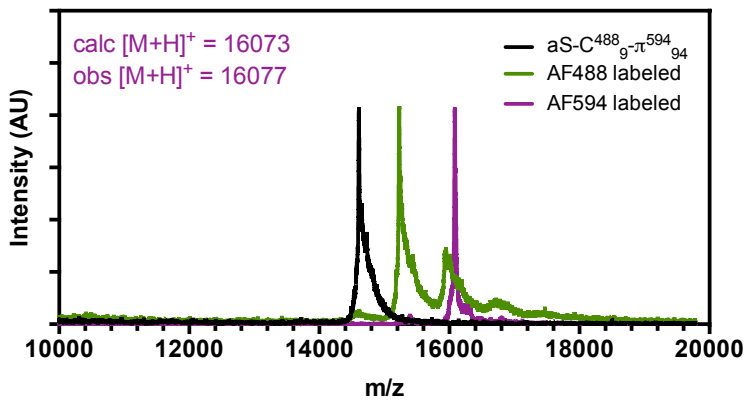

(B)
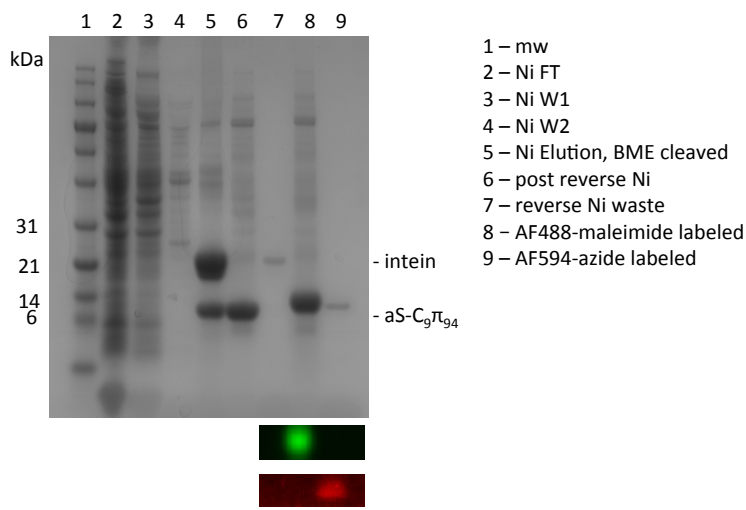

Figure S21. Recombinant $\boldsymbol{\alpha S}-C^{\mathbf{4 8 8}} \boldsymbol{\pi}^{\mathbf{5 9 4}}{ }_{\mathbf{9 4}}$ (A) MALDI-MS (B) $15 \%$ polyacrylamide SDS-PAGE gel (Coomassie stain) and FITC and cy5 fluorescence filters 
(B)
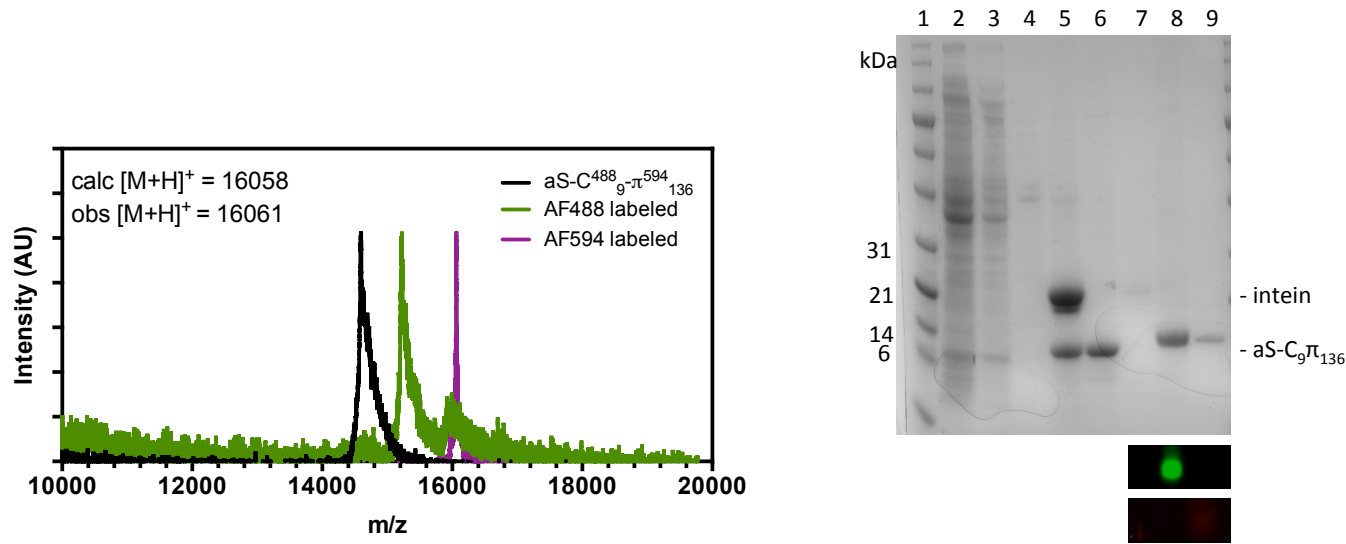

$1-\mathrm{mw}$

2- Ni FT

$3-\mathrm{Ni}$ W

4-Ni W2

5 - Ni Elution, BME cleaved

- post reverse $\mathrm{N}$

7 - reverse Ni waste

- AF488-maleimide labeled

9-AF594-azide labeled

Figure S22. Recombinant $\boldsymbol{\alpha S}-\mathbf{C}^{\mathbf{4 8 8}} \boldsymbol{9}^{\mathbf{5 9 4}}{ }_{\mathbf{1 3 6}}$ (A) MALDI-MS (B) $15 \%$ polyacrylamide SDS-PAGE gel (Coomassie stain) and FITC and cy5 fluorescence filters

(A)

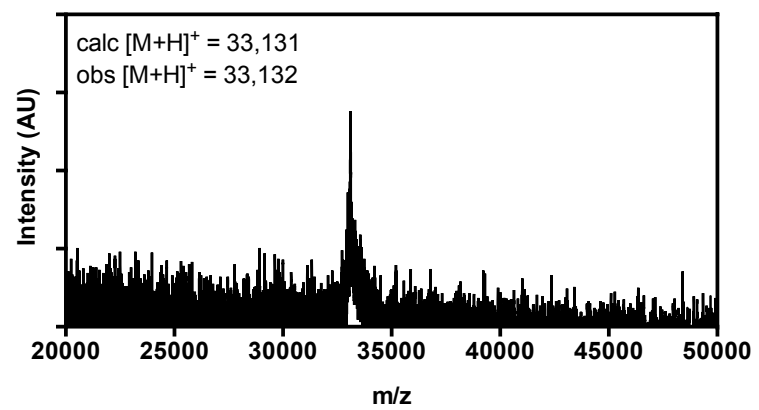

(C)

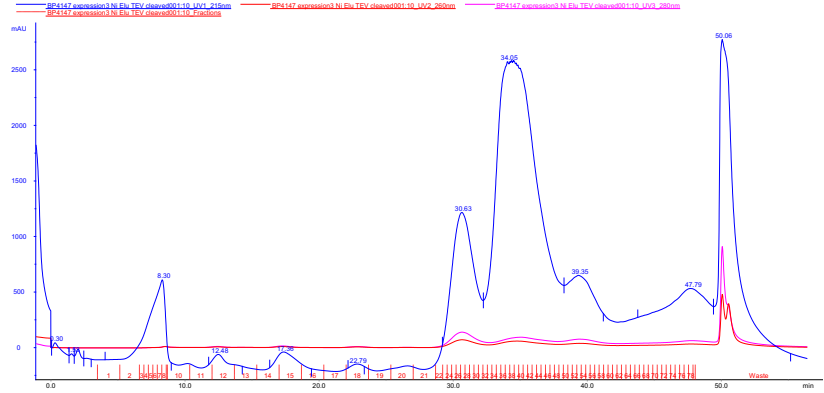

(B)

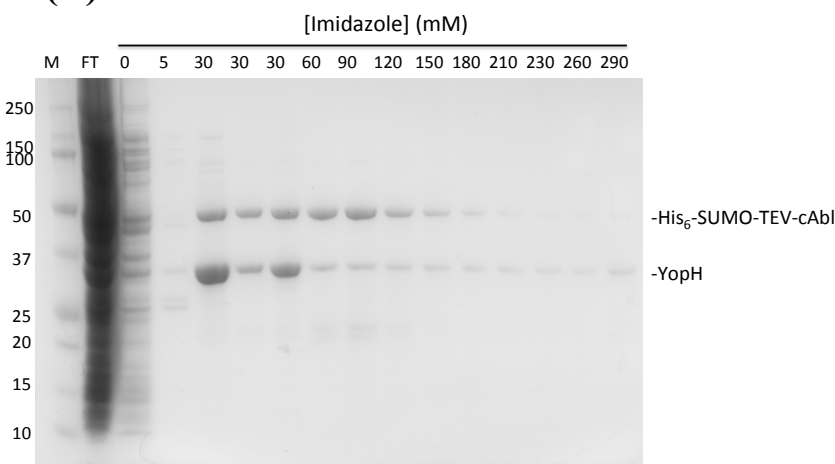

(D)

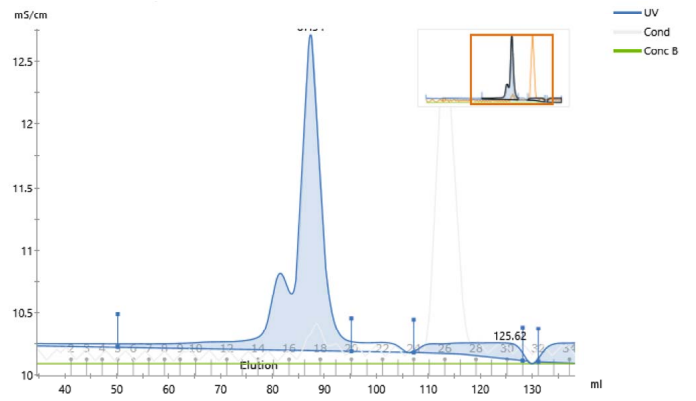

Figure S23. Recombinant cAbl kinase (A) MALDI-MS (B) 15\% polyacrylamide SDS-PAGE gel (Coomassie stain) (C) FPLC, HiTrap Q 5 mL (D) Size exclusion chromatography, Superdex 75 
(A)

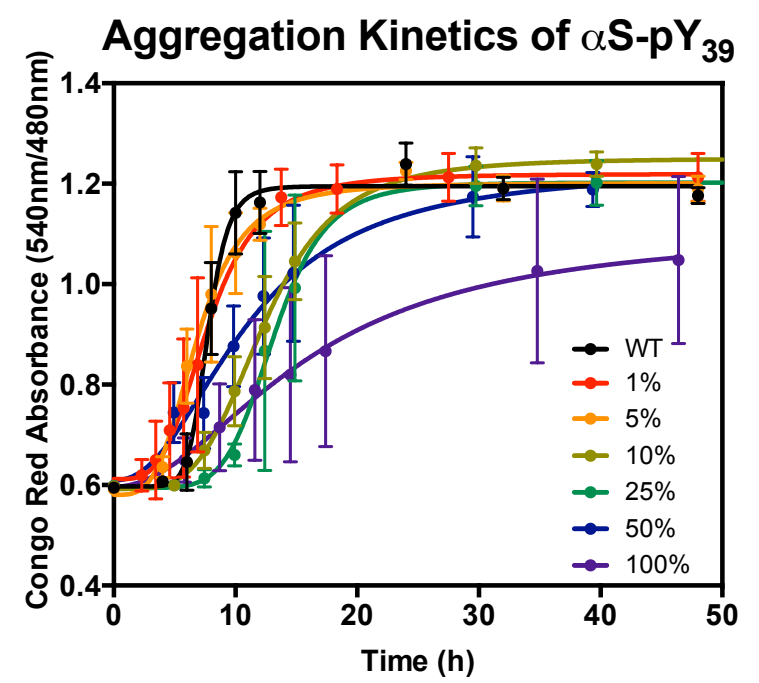

(B)

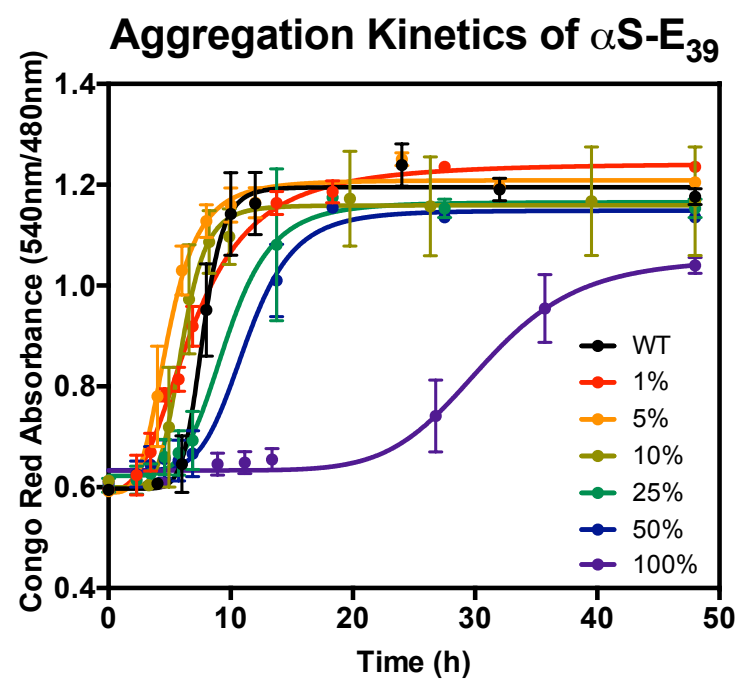

Figure S24. Aggregation kinetics by Congo Red. Aggregation kinetics of (A) $\alpha \mathrm{S}-\mathrm{p} \mathrm{Y}_{39}$ and (B) $\alpha \mathrm{S}$ phosphorylation mimic $\mathrm{E}_{39}$ at various percentages of monomeric starting material.

(A)
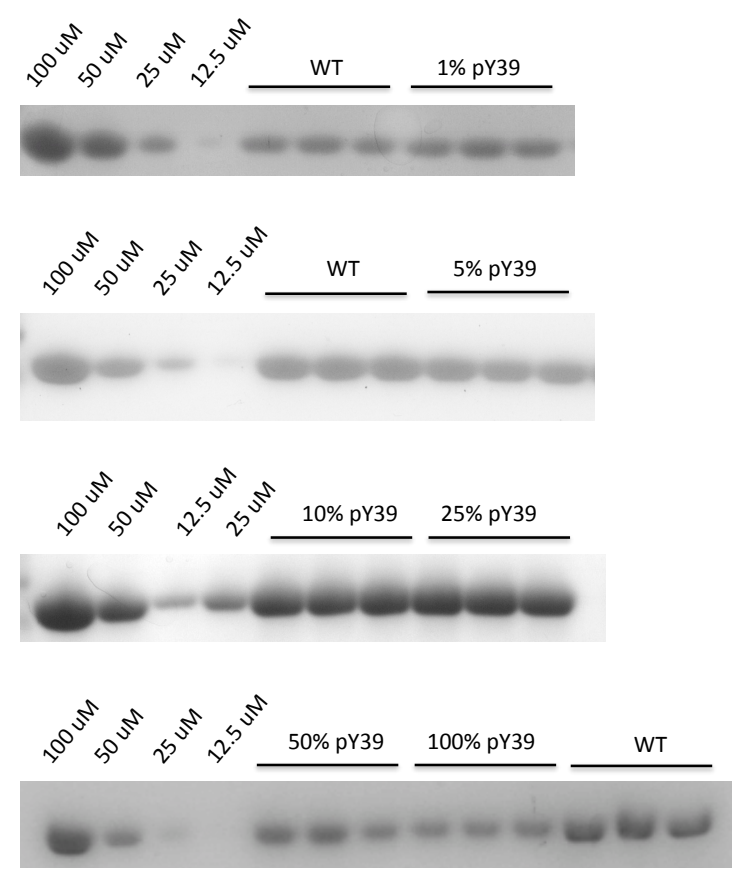

(B)

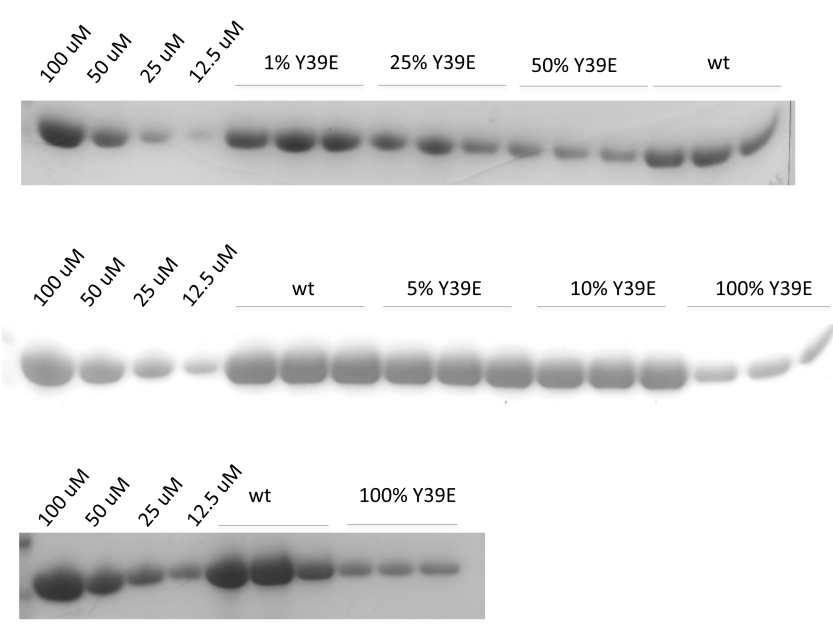

Figure S25. Fibril incorporation assay by SDS-PAGE (A) Percentage incorporation assay for $\alpha \mathrm{S}-$ $\mathrm{pY}_{39}$ and (B) $\alpha \mathrm{S}$ phosphorylation mimic Y39E (E 39 ). Standards: $100 \mu \mathrm{M}, 50 \mu \mathrm{M}, 25 \mu \mathrm{M}$, and 12.5 $\mu \mathrm{M}$ wild type $\alpha \mathrm{S}$ monomer. 
(A)
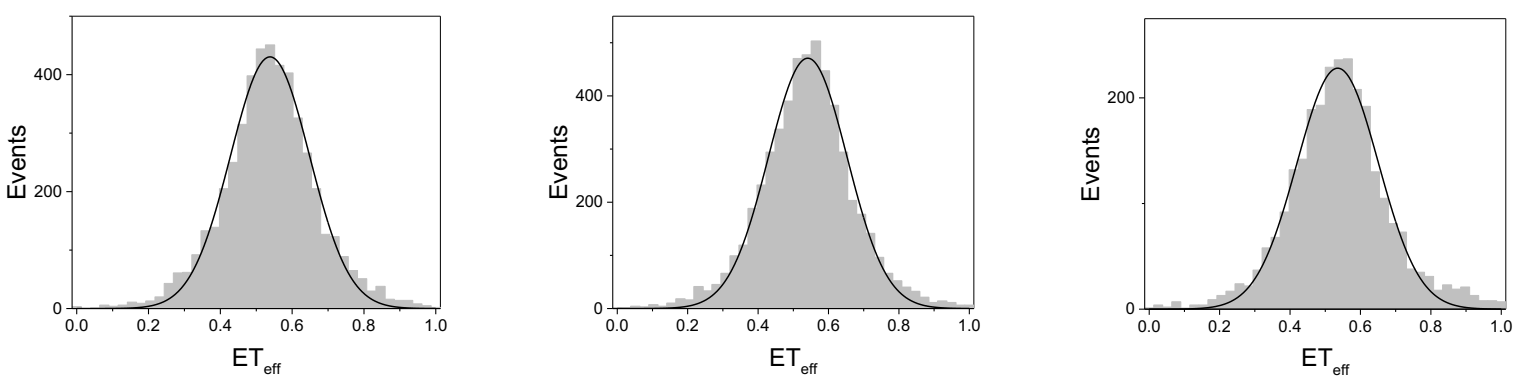

(B)
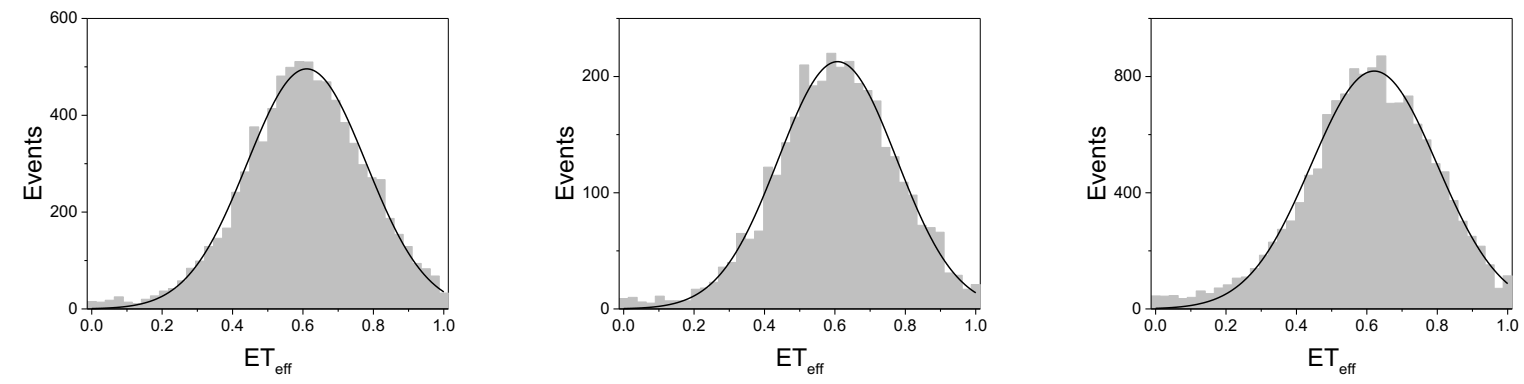

(C)
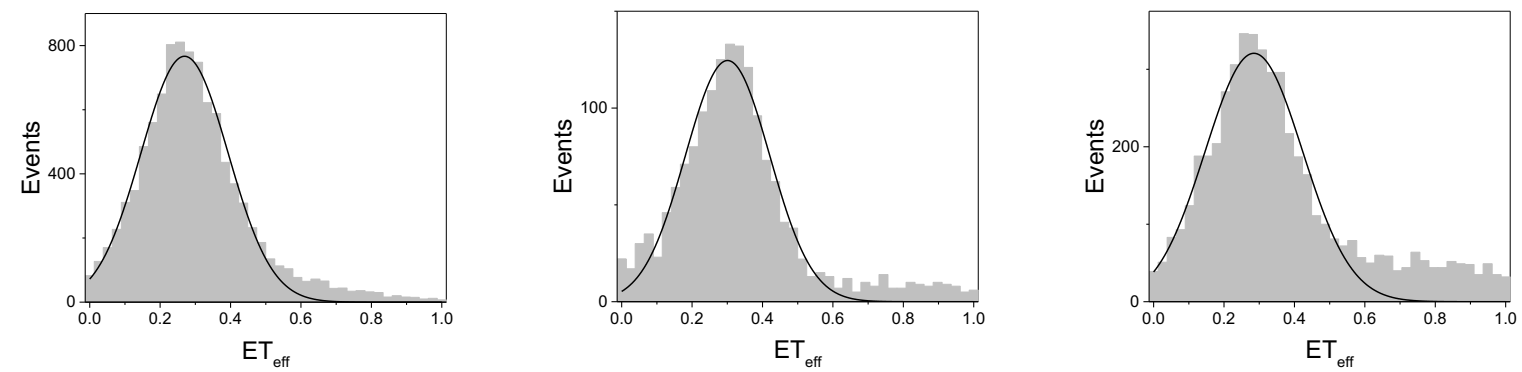

(D)
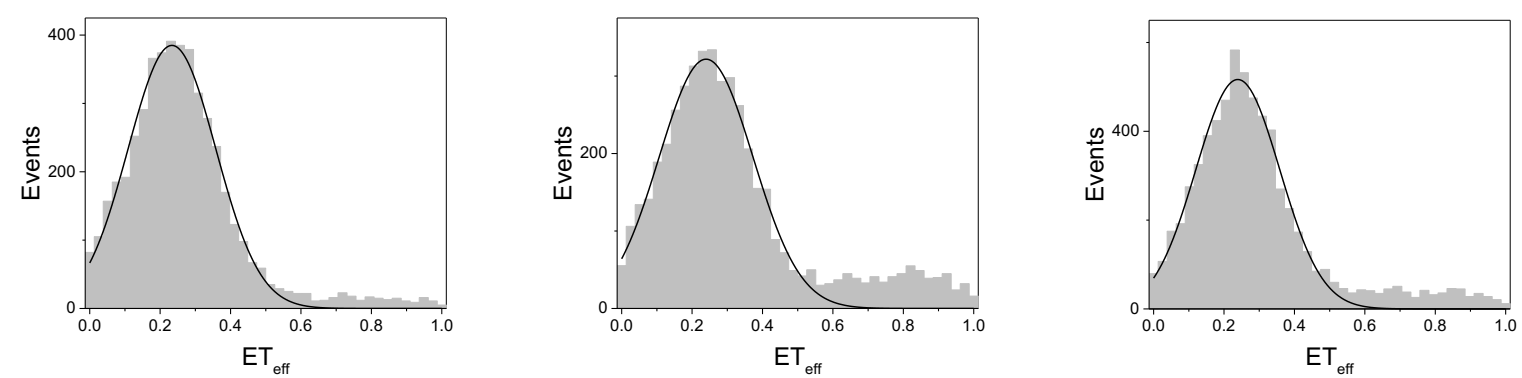

Figure S26. smFRET histograms comparing $\alpha \mathrm{S}$ WT and $\mathrm{pY}_{39}$ conformation in solution. (A) $\alpha \mathrm{S}-$ $\mathrm{C}^{488}{ }_{9} \pi^{594}{ }_{72}$ control (B) $\alpha \mathrm{S}-\mathrm{C}^{488}{ }_{9} \mathrm{pY}_{39} \pi^{594}{ }_{72}$ (C) $\alpha \mathrm{S}-\mathrm{C}^{488}{ }_{9} \pi^{594}{ }_{136}$ control (D) $\alpha \mathrm{S}-\mathrm{C}^{488}{ }_{9} \mathrm{pY} \mathrm{Y}_{39} \pi^{594}{ }_{136}$. All data were fit to Gaussian distributions. 
Table S1. smFRET data

\begin{tabular}{|l|c|c|c|c|}
\hline & \multicolumn{3}{|c|}{$\mathrm{ET}_{\text {eff }}$ center } & Mean $\mathrm{ET}_{\text {eff }}$ center \\
\hline$\alpha \mathrm{S}-\mathrm{C}^{488}{ }_{9} \pi^{594}{ }_{72}$ & 0.53839 & 0.54107 & 0.53548 & 0.53831 \\
\hline$\alpha \mathrm{S}-\mathrm{C}^{488}{ }_{9} \mathrm{pY}_{39} \pi^{594}{ }_{72}$ & 0.61061 & 0.60744 & 0.60289 & 0.60698 \\
\hline$\alpha \mathrm{S}^{4} \mathrm{C}^{488}{ }_{9} \pi^{594}{ }_{136}$ & 0.26932 & 0.30078 & 0.28484 & 0.28498 \\
\hline$\alpha \mathrm{S}-\mathrm{C}^{488}{ }_{9} \mathrm{pY}_{39} \pi^{594}{ }_{136}$ & 0.23354 & 0.23976 & 0.23914 & 0.23748 \\
\hline
\end{tabular}

\begin{tabular}{|l|c|c|c|c|}
\hline & \multicolumn{3}{|c|}{ width } & Mean width \\
\hline$\alpha \mathrm{S}-\mathrm{C}^{488}{ }_{9} \pi^{594}{ }_{72}$ & 0.22039 & 0.22307 & 0.23131 & 0.22492 \\
\hline$\alpha \mathrm{S}-\mathrm{C}^{488}{ }_{9} \mathrm{pY}_{39} \pi^{594}{ }_{72}$ & 0.33848 & 0.33597 & 0.35806 & 0.34417 \\
\hline$\alpha \mathrm{S}-\mathrm{C}^{488}{ }_{9} \pi^{594}{ }_{136}$ & 0.24760 & 0.23940 & 0.27646 & 0.25449 \\
\hline$\alpha \mathrm{S}-\mathrm{C}^{488}{ }_{9} \mathrm{pY}_{39} \pi^{594}{ }_{136}$ & 0.24929 & 0.26698 & 0.23869 & 0.25165 \\
\hline
\end{tabular}
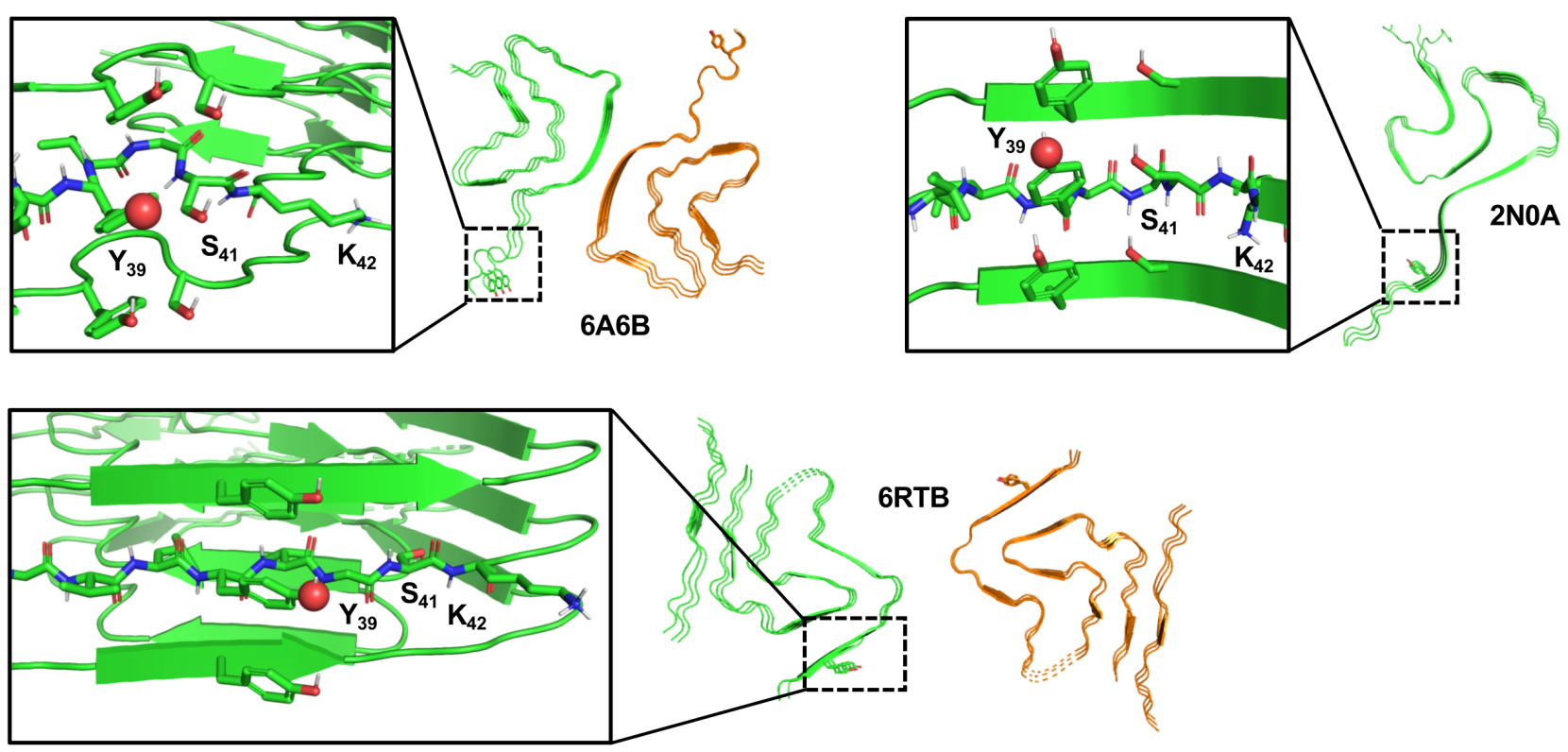

Figure S27. Potential interactions of $\mathbf{p Y}_{39}$ in fibrils. Cryo-EM structures (PDB ID: 6A6B and 6RTB) and solid state NMR structure (PDB ID: 2NOA) showing Y 39 viewed down fibril axis or sideon (insets). ${ }^{10-12}$ 

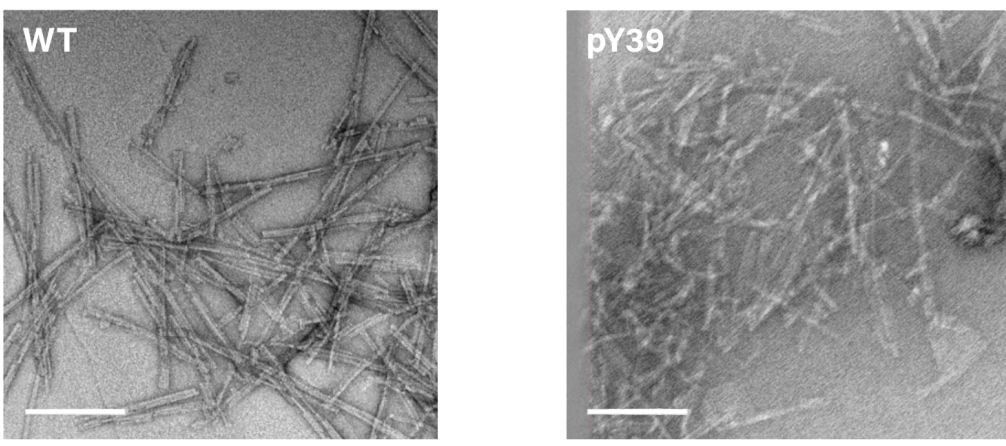

Figure S28. Transmission Electron Microscopy images of pY39 fibrils. TEM images of fibrils formed from $100 \% \mathrm{WT}$ in comparison with those comprised of $25 \% \mathrm{pY}_{39}$. Scale bars indicate 200 $\mathrm{nm}$.

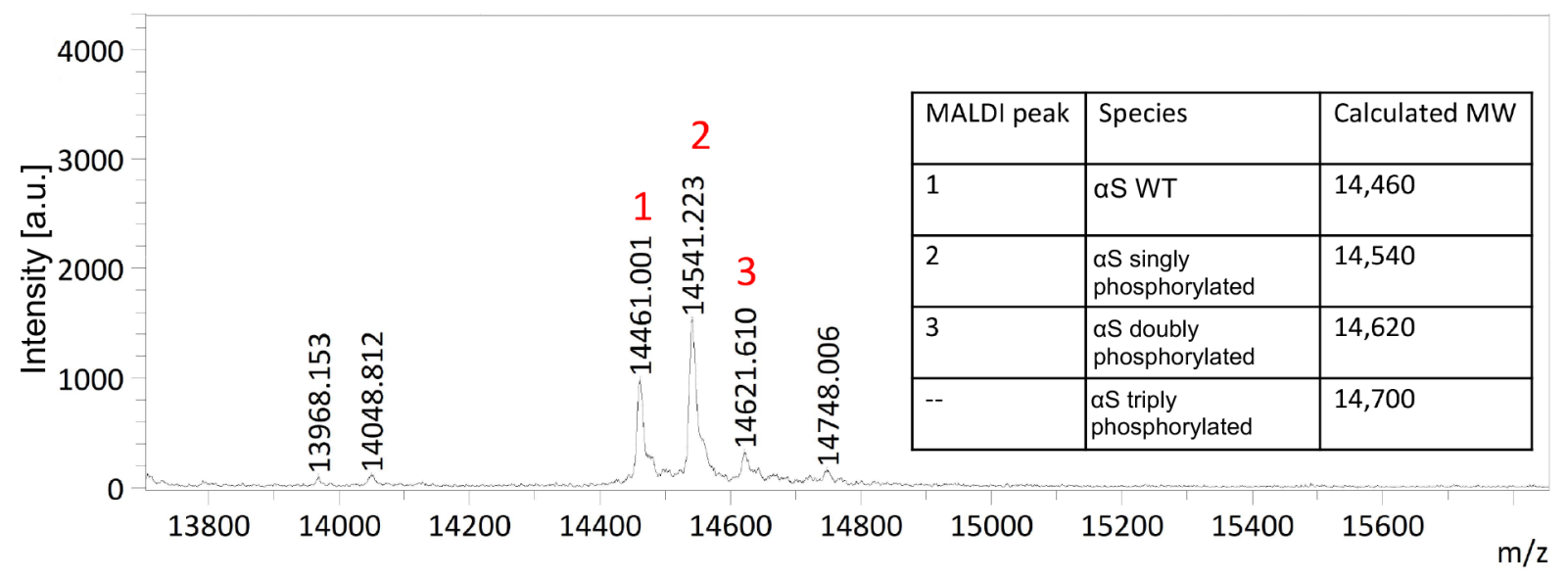

Figure S29. Multiple phosphorylation of $\alpha \mathrm{S}$ full-length by c-Abl. MALDI-MS of WT $\alpha \mathrm{S}$ fulllength after $2 \mathrm{~h}$ in vitro reaction with c-Abl enzyme. Appearance of doubly phosphorylated species is observed prior to completion of single phosphorylation. 


\section{References}

[1] Batjargal, S., Walters, C. R., and Petersson, E. J. (2015) Inteins as Traceless Purification Tags for Unnatural Amino Acid Proteins, J. Am. Chem. Soc. 137, 1734-1737.

[2] Haney, C. M., Wissner, R. F., Warner, J. B., Wang, Y. X. J., Ferrie, J. J., Covell, D. J., Karpowicz, R. J., Lee, V. M. Y., and Petersson, E. J. (2016) Comparison of Strategies for NonPerturbing Labeling of Alpha-Synuclein to Study Amyloidogenesis, Org. Biomol. Chem. 14, 1584-1592.

[3] Xiao, Q., Zhang, F. R., Nacev, B. A., Liu, J. O., and Pei, D. H. (2010) Protein N-Terminal Processing: Substrate Specificity of Escherichia Coli and Human Methionine Aminopeptidases, Biochemistry 49, 5588-5599.

[4] Liu, J. J., Chan, K. K. J., and Chan, W. (2016) Identification of Protein Thiazolidination as a Novel Molecular Signature for Oxidative Stress and Formaldehyde Exposure, Chem. Res. Toxicol. 29, 1865-1871.

[5] Bang, D., and Kent, S. B. H. (2004) A One-Pot Total Synthesis of Crambin, Angew. Chem.-Int. Edit. 43, 2534-2538.

[6] Merrifield, R. B. (1963) Solid Phase Peptide Synthesis .1. Synthesis of a Tetrapeptide, J. Am. Chem. Soc. 85, 2149-2154.

[7] Albanese, S. K., Parton, D. L., Isik, M., Rodriguez-Laureano, L., Hanson, S. M., Behr, J. M., Gradia, S., Jeans, C., Levinson, N. M., Seeliger, M. A., and Chodera, J. D. (2018) An Open Library of Human Kinase Domain Constructs for Automated Bacterial Expression, Biochemistry 57, 4675-4689.

[8] Fang, G. M., Li, Y. M., Shen, F., Huang, Y. C., Li, J. B., Lin, Y., Cui, H. K., and Liu, L. (2011) Protein Chemical Synthesis by Ligation of Peptide Hydrazides, Angew. Chem.-Int. Edit. 50, 7645-7649.

[9] Muir, T. W., Sondhi, D., and Cole, P. A. (1998) Expressed Protein Ligation: A General Method for Protein Engineering, Proc. Natl. Acad. Sci. U. S. A. 95, 6705-6710.

[10] Tuttle, M. D., Comellas, G., Nieuwkoop, A. J., Covell, D. J., Berthold, D. A., Kloepper, K. D., Courtney, J. M., Kim, J. K., Barclay, A. M., Kendall, A., Wan, W., Stubbs, G., Schwieters, C. D., Lee, V. M. Y., George, J. M., and Rienstra, C. M. (2016) Solid-State Nmr Structure of a Pathogenic Fibril of Full-Length Human Alpha-Synuclein, Nat. Struct. Mol. Biol. 23, 409415.

[11] Guerrero-Ferreira, R., Taylor, N. M. I., Mona, D., Ringler, P., Lauer, M. E., Riek, R., Britschgi, M., and Stahlberg, H. (2018) Cryo-Em Structure of Alpha-Synuclein Fibrils, eLife 7, e36402.

[12] Li, Y., Zhao, C., Luo, F., Liu, Z., Gui, X., Luo, Z., Zhang, X., Li, D., Liu, C., and Li, X. (2018) Amyloid Fibril Structure of A-Synuclein Determined by Cryo-Electron Microscopy, Cell Res. 28, 897-903. 